TRANSACTIONS OF THE

AMERICAN MATHEMATICAL SOCIETY

Volume 359, Number 10, October 2007, Pages 4787-4828

S 0002-9947(07)04022-6

Article electronically published on May 17, 2007

\title{
GENERALIZATIONS OF CHEBYSHEV POLYNOMIALS AND POLYNOMIAL MAPPINGS
}

\author{
YANG CHEN, JAMES GRIFFIN, AND MOURAD E.H. ISMAIL
}

\begin{abstract}
In this paper we show how polynomial mappings of degree $\mathfrak{K}$ from a union of disjoint intervals onto $[-1,1]$ generate a countable number of special cases of generalizations of Chebyshev polynomials. We also derive a new expression for these generalized Chebyshev polynomials for any genus $g$, from which the coefficients of $x^{n}$ can be found explicitly in terms of the branch points and the recurrence coefficients. We find that this representation is useful for specializing to polynomial mapping cases for small $\mathfrak{K}$ where we will have explicit expressions for the recurrence coefficients in terms of the branch points. We study in detail certain special cases of the polynomials for small degree mappings and prove a theorem concerning the location of the zeroes of the polynomials. We also derive an explicit expression for the discriminant for the genus 1 case of our Chebyshev polynomials that is valid for any configuration of the branch point.
\end{abstract}

\section{INTRODUCTION AND PRELIMINARIES}

Akhiezer 2], 1] and, Akhiezer and Tomčuk [3] introduced orthogonal polynomials on two intervals which generalize the Chebyshev polynomials. He observed that the study of properties of these polynomials requires the use of elliptic functions. In the case of more than two intervals, Tomčuk [17, investigated their Bernstein-Szegö asymptotics, with the theory of Hyperelliptic integrals, and found expressions in terms of a certain Abelian integral of the third kind. However, in his formulation, certain unknown points on a Hyperelliptic Riemann surface emerge due to the lack of an explicit representation of the original polynomials. This was circumvented in [9]. In his book on elliptic functions [4] Akhiezer obtained explicit formulas for the two interval cases as an example of the application of elliptic functions to the theory of conformal mapping.

In 1984 Al-Salam, Allaway, and Askey introduced sieved ultraspherical polynomials which are orthogonal with respect to an absolutely continuous measure supported on $[-1,1]$ but the weight function vanishes at $k+1$ points. Ismail [12] observed that the vanishing of the weight function means that the polynomials are orthogonal on several adjacent intervals. He then introduced one additional parameter in the definition of the sieved ultraspherical polynomials which made them orthogonal on several intervals with gaps. In particular his polynomials include analogues of the Chebyshev polynomials of the first and second kind. Their continuous spectrum is $T_{k}^{-1}([-c, c])$, for $c \in(-1,1)$, where $T_{k}$ is a Chebyshev polynomial

Received by the editors January 27, 2004 and, in revised form, May 3, 2005.

2000 Mathematics Subject Classification. Primary 33C45.

(C)2007 American Mathematical Society 
of the first kind. Moreover these polynomials have simple closed form expressions and elementary generating functions. More general sieved polynomials are in 7 .

In [14] a study of Chebyshev type polynomials was carried out. They arose as extremal polynomials and are orthogonal on several intervals. The Chebyshev type polynomials satisfy similar extremal properties to the classical Chebyshev polynomials on $[-1,1]$. In particular they deviate least from zero on a union of disjoint intervals. The extremal polynomials also have the property that they are orthogonal with respect to some weight function of the form

$$
\frac{\pi_{g-1}(x)}{\sqrt{\prod_{j=1}^{2 g}\left(x-a_{j}\right)}}, \quad x \in E,
$$

where $E:=\bigcup_{j=1}^{2 g-1}\left(a_{j}, a_{j+1}\right)$, and the polynomial $\pi_{g-1}(x)$ is chosen such that the weight is positive on the interior of $E$. The existence of an extremal polynomial on any set $E$ is clearly not guaranteed. It was shown in [14 that the existence of an extremal polynomial on a set $E$ is equivalent to the asymptotic periodicity of the recurrence coefficients of a sequence of orthogonal polynomials on $E$. The extremal polynomial also has the property that it maps the set $E$ onto $[-1,1]$.

In [10, using a polynomial mapping of this kind, a prescription was given for the construction of a sequence of orthogonal polynomials on $E$ from a sequence orthogonal on a single interval. The corresponding weight supported on $E$ can be obtained using the following procedure, outlined in 10 . In this generality closed form expressions and generating functions are not available. This raises the question of finding a class of polynomials as general as possible which has the extremal properties of the polynomials in Peherstorfer 14 and the simple structural formulas of Ismail's polynomials in [12. The first step was carried out in [9] and is further developed here. In the present paper we develop this work further by borrowing ideas from polynomial mappings and sieved polynomials.

We start by choosing a polynomial of degree $\mathfrak{K}-1$ and call this polynomial $W_{\mathfrak{K}-1}(t)$. Then we form the expression

$$
\frac{W_{\mathfrak{K}-1}(t)}{M_{\mathfrak{K}}(t)-x}=\frac{W_{\mathfrak{K}-1}(t)}{l_{\mathfrak{K}} \prod_{i=1}^{\mathfrak{K}}\left(t-M_{i}^{-1}(x)\right)}=\sum_{i=1}^{\mathfrak{K}} \frac{\omega_{i}(x)}{t-M_{i}^{-1}(x)}
$$

where the $M_{i}^{-1}(x)$ are a complete set of inverse branches of $M_{\mathfrak{K}}(x)$ and $l_{\mathfrak{K}}$ is the leading coefficient of $M_{\mathfrak{K}}(t)$. Rearranging the above equation and taking the limit as $t \rightarrow M_{i}^{-1}(x)$ we find

$$
\omega_{i}(x)=\frac{W_{\mathfrak{K}-1}\left(M_{i}^{-1}(x)\right)}{M_{\mathfrak{K}}^{\prime}\left(M_{i}^{-1}(x)\right)} .
$$

By comparing coefficients of $t^{\mathfrak{K}-1}$ in (1.1) it is clear that

$$
\sum_{j=1}^{\mathfrak{K}} \omega_{j}(x)=c
$$

where $c$ is the quotient of the leading coefficients of $W_{\mathfrak{K}-1}(x)$ and $M_{\mathfrak{K}}(x)$. So, having chosen a particular polynomial $W_{\mathfrak{K}-1}(x)$ we are in a position to define the transformed weight function in the following way. Let $E_{0}$ be the interval $[a, b]$, and 
$P_{n}(x)$ the polynomials orthogonal with respect to $w_{0}(x)$ over $E_{0}$ :

$$
\begin{aligned}
h_{n} \delta_{n m} & =\int_{a}^{b} P_{n}(x) P_{m}(x) w_{0}(x) d x \\
& =\frac{1}{c} \int_{a}^{b} P_{n}(x) P_{m}(x)\left(\sum_{j=1}^{\mathfrak{K}} \omega_{j}(x)\right) w_{0}(x) d x \\
& =\frac{1}{c} \sum_{j=1}^{\mathfrak{K}} \int_{A_{j}} P_{n}\left(M_{\mathfrak{K}}(t)\right) P_{m}\left(M_{\mathfrak{K}}(t)\right) \frac{W_{\mathfrak{K}-1}(t)}{M_{\mathfrak{K}}^{\prime}(t)} M_{\mathfrak{K}}^{\prime}(t) w_{0}\left(M_{\mathfrak{K}}(t)\right) d t \\
& =\frac{1}{c} \int_{E} P_{n}\left(M_{\mathfrak{K}}(t)\right) P_{m}\left(M_{\mathfrak{K}}(t)\right) W_{\mathfrak{K}-1}(t) w_{0}(t) d t,
\end{aligned}
$$

where

$$
E:=\bigcup_{j=1}^{\mathfrak{K}} A_{j}
$$

and

$$
M_{j}^{-1}:[a, b] \rightarrow A_{j} .
$$

So the new sequence of polynomials is orthogonal on the disjoint set $E$ with respect to the measure

$$
\widehat{w}(t) d t=W_{\mathfrak{K}-1}(t) w_{0}\left(M_{\mathfrak{K}}(t)\right) .
$$

It was shown in [10] that

$$
\widehat{P}_{n \mathfrak{K}}(x)=P_{n}\left(M_{\mathfrak{K}}(x)\right), \quad n=0,1,2, \ldots
$$

This generates every $\mathfrak{K}^{\text {th }}$ polynomial in the sequence. The polynomials $\widehat{P}_{n \mathfrak{K}+j}(x)$, $1 \leq j \leq \mathfrak{K}-1$, are referred to as the intermediate polynomials. Formulas relating the intermediate polynomials and recurrence coefficients of the new sequence to known quantities of the old sequence are given in 10 .

Some explicit expressions for the Chebyshev polynomials on several intervals, along with many other important results, can be found in [9]. This paper is organized as follows. In this section we recall the theta function representation of these polynomials which was derived in [9]. We also present some facts concerning the polynomials that will be referred to throughout this paper.

Section 2 is a study of two auxiliary polynomials $S_{g}(x ; n)$ and $G_{g+1}(x ; n)$, of degree $g$ and $g+1$ respectively. The auxiliary polynomials are fundamental to the theory of the generalization of Chebyshev polynomials under consideration. We also derive for the first time non-linear difference equations satisfied by the recurrence coefficients that appear in the three term recurrence relation.

In section 3 we derive a product representation for our polynomials from which one can obtain the coefficients of the polynomial in terms of the recurrence coefficients and the branch points. This representation is a new one that involves the auxiliary polynomials studied in section 2 . It is particularly useful when we consider certain configurations of the branch points where the recurrence coefficients simplify into an algebraic form.

In section 4 of this paper we show how certain polynomial mappings give rise to a countable number of special cases of our generalization of the Chebyshev polynomials. These cases correspond to when the recurrence coefficients are periodic. We 
give explicit formulas for the polynomials of degree not equal to an integer multiple of the period.

The monic form of the Chebyshev polynomials on several intervals will be denoted by $P_{n}(x)$. They are orthogonal on $E:=[-1, \alpha] \cup_{j=1}^{g-1}\left[\beta_{j}, \alpha_{j+1}\right] \cup\left[\beta_{g}, 1\right]$ with respect to the following weight:

$$
w(x)=\frac{1}{\pi} \sqrt{\frac{\prod_{j=1}^{g}\left(x-\alpha_{j}\right)}{\left(1-x^{2}\right) \prod_{j=1}^{g}\left(x-\beta_{j}\right)}},
$$

so that

$$
\int_{E} P_{n}(x) P_{m}(x) w(x) d x=\delta_{n m} h_{n}
$$

The polynomials of the second kind are

$$
Q_{n}(x)=\int_{E} \frac{P_{n}(x)-P_{n}(t)}{x-t} w(t) d t .
$$

Both $P_{n}(x)$ and $Q_{n}(x)$ satisfy the recurrence relation

$$
x u_{n}(x)=u_{n+1}(x)+b_{n+1} u_{n}(x)+a_{n} u_{n-1}(x) ;
$$

see [2]. The Stieltjes transform of the weight function is

$$
\psi(z):=\int_{E} \frac{w(t) d t}{z-t}=\sqrt{\frac{\prod_{j=1}^{g}\left(z-\alpha_{j}\right)}{\left(z^{2}-1\right) \prod_{j=1}^{g}\left(z-\beta_{j}\right)}}, \quad z \notin E .
$$

Note that

$$
\psi(x)=\frac{\pi}{i} w(x)
$$

In [9], Chen and Lawrence considered a hyperelliptic Riemann surface of genus $g$,

$$
y^{2}(z)=\left(z^{2}-1\right) \prod_{j=1}^{g}\left(z-\alpha_{j}\right)\left(z-\beta_{j}\right),
$$

from which a representation for the polynomials in terms of the associated Riemann theta function was constructed. They also gave a representation for the recurrence coefficients in terms of the theta functions. To formulate these representations the following facts regarding this Riemann surface are required.

A canonical basis of cycles is chosen on the surface that is composed of $2 g$ closed contours. We denote by the a-cycles the $g$ closed contours that lie on one sheet of the surface, and the b-cycles to be the remaining $g$ contours that travel over both sheets. For a surface of genus $g$ a basis of holomorphic differentials can be written in terms of $g$ linearly independent differentials,

$$
\left\{\frac{d x}{y(x)}, \frac{x d x}{y(x)}, \ldots, \frac{x^{g-1} d x}{y(x)}\right\} .
$$

If we let

$$
A_{j k}=\int_{a_{k}} \frac{x^{g-j}}{y(x)} d x
$$

then the $d \omega_{j}$ as defined,

$$
d \omega_{j}=\sum_{j=1}^{g}\left(A^{-1}\right)_{j k} \frac{x^{g-j}}{y(x)} d x
$$


form an orthonormal basis such that

$$
\int_{a_{j}} d \omega_{k}=\delta_{j k}
$$

We can then define the period matrix $B$ whose entries are

$$
B_{j k}=\int_{b_{j}} d \omega_{k} .
$$

The Riemann theta function associated with the surface is defined to be

$$
\vartheta(s ; B)=\vartheta(s):=\sum_{t \in \mathbb{Z}^{g}} \exp (i \pi(t, B t)+2 \pi i(t, s)),
$$

where $s$ is a $g$-vector, and $(u, v)$ denotes the standard scalar product of two vectors. Convergence of the series is assured since $\Im B$ is a positive definite matrix. The theta function has the following properties. For $t \in \mathbb{Z}^{g}$,

$$
\begin{aligned}
& \vartheta(-s)=\vartheta(s), \\
& \vartheta(s+t)=\vartheta(s), \\
& \vartheta(s+B t)=e^{-i \pi[(t, B t)+2(t, s)]} \vartheta(s) .
\end{aligned}
$$

Both the polynomials and the recurrence coefficients are given below. The expressions are taken from [9]. However, in view of lemma 10.2 in [9] and the quasiperiodicity of the theta functions we have simplified the expressions slightly:

$$
P_{n}(x)=\frac{1}{2}\left(\mathcal{E}_{n}\left(\mathfrak{p}_{x}\right)+\tilde{\mathcal{E}}_{n}\left(\mathfrak{p}_{x}\right)\right),
$$

where, for $n \geq 1$,

$$
\mathcal{E}_{n}\left(\mathfrak{p}_{x}\right)=2 e^{-n\left(\chi_{0}+\Omega\left(\mathfrak{p}_{x}\right)\right)} \frac{\vartheta\left(\mathbf{u}^{+}\right) \vartheta\left(\mathbf{u}_{x}-n \hat{\mathbf{B}}\right)}{\vartheta\left(\mathbf{u}^{+}+n \hat{\mathbf{B}}\right) \vartheta\left(\mathbf{u}_{x}\right)}
$$

and

$$
\tilde{\mathcal{E}}_{n}\left(\mathfrak{p}_{x}\right)=2 e^{n\left(\Omega\left(\mathfrak{p}_{x}\right)-\chi_{0}\right)} \frac{\vartheta\left(\mathbf{u}^{+}\right) \vartheta\left(\mathbf{u}_{x}+n \hat{\mathbf{B}}\right)}{\vartheta\left(\mathbf{u}^{+}+n \hat{\mathbf{B}}\right) \vartheta\left(\mathbf{u}_{x}\right)} .
$$

Using this notation,

$$
Q_{n}(x)=\frac{\psi(x)}{2}\left(\tilde{\mathcal{E}}_{n}\left(\mathfrak{p}_{x}\right)-\mathcal{E}_{n}\left(\mathfrak{p}_{x}\right)\right) .
$$

Here, $\mathfrak{p}_{x}$ is a point on the top sheet of the Riemann surface that corresponds to a point $x$ in the complex plane, and

$$
\begin{aligned}
\Omega\left(\mathfrak{p}_{x}\right) & =\int_{\mathfrak{p}_{1}}^{\mathfrak{p}_{x}} d \Omega \\
& :=\int_{\mathfrak{p}_{1}}^{\mathfrak{p}_{x}} \frac{t^{g}+\sum_{j=0}^{g-1} k_{j} t^{j}}{y(t)} d t .
\end{aligned}
$$

The $k_{j}$ can be determined from the condition that

$$
\int_{a_{j}} d \Omega=0, \quad j=1, \ldots, g .
$$

$\mathbf{u}_{x}$ is defined to be a g-vector,

$$
\mathbf{u}_{x}:=\left(\int_{p_{1}}^{p_{x}} d \omega_{1}, \ldots, \int_{p_{1}}^{p_{x}} d \omega_{g}\right)^{T}
$$


and $\mathbf{u}^{ \pm}:=\mathbf{u}_{\infty^{ \pm}}$. If $\hat{\mathbf{B}}$ is the vector whose components are

$$
\hat{\mathbf{B}}_{j}=\frac{1}{2 \pi i} \int_{b_{j}} d \Omega, \quad j=1, \ldots, g
$$

then

$$
\hat{\mathbf{B}} \equiv \mathbf{u}^{+}-\mathbf{u}^{-}\left(\bmod \mathbb{Z}^{g}\right)
$$

Finally, the equilibrium potential of the set $E$ is

$$
\chi_{0}=\int_{\mathfrak{p}_{1}}^{\infty^{+}}\left(\frac{t^{g}+\sum_{j=0}^{g} k_{j} t^{j}}{y(t)}-\frac{1}{t}\right) d t .
$$

With this notation, we have the following expressions for the recurrence coefficients:

$$
a_{n}= \begin{cases}2 e^{-2 \chi_{0} \frac{\vartheta\left(\mathbf{u}^{+}-\hat{\mathbf{B}}\right)}{\vartheta\left(\mathbf{u}^{+}+\hat{\mathbf{B}}\right)}} & \text { if } n=1, \\ e^{-2 \chi_{0} \frac{\vartheta\left(\mathbf{u}^{+}+(n-1) \hat{\mathbf{B}}\right) \vartheta\left(\mathbf{u}^{+}-n \hat{\mathbf{B}}\right)}{\vartheta\left(\mathbf{u}^{+}-(n-1) \hat{\mathbf{B}}\right) \vartheta\left(\mathbf{u}^{+}+n \hat{\mathbf{B}}\right)}} & \text { if } n>1\end{cases}
$$

and

$$
b_{n}=\frac{1}{2} \sum_{j=1}^{g}\left(\beta_{j}-\alpha_{j}\right)+\sum_{j=1}^{g}\left(A^{-1}\right)_{j 1}\left[\frac{\vartheta_{j}^{\prime}\left(\mathbf{u}^{+}+\hat{\mathbf{B}}\right)}{\vartheta\left(\mathbf{u}^{+}+\mathbf{B}\right)}-\frac{\vartheta_{j}^{\prime}\left(\mathbf{u}^{+}\right)}{\vartheta\left(\mathbf{u}^{+}\right)}+\frac{\vartheta_{j}^{\prime}\left(\mathbf{u}^{+}+(n-1) \hat{\mathbf{B}}\right)}{\vartheta\left(\mathbf{u}^{+}+(n-1) \hat{\mathbf{B}}\right)}-\frac{\vartheta_{j}^{\prime}\left(\mathbf{u}^{+}+n \hat{\mathbf{B}}\right)}{\vartheta\left(\mathbf{u}^{+}+n \hat{\mathbf{B}}\right)}\right] .
$$

Here,

Also,

$$
\vartheta_{j}^{\prime}(\mathbf{u}):=\frac{\partial}{\partial u_{j}} \vartheta(\mathbf{u})
$$

$$
h_{n}=2 e^{-2 n \chi_{0}} \frac{\vartheta\left(\mathbf{u}^{+}-n \hat{\mathbf{B}}\right)}{\vartheta\left(\mathbf{u}^{+}+n \hat{\mathbf{B}}\right)} .
$$

Theorem 1.1. The polynomials of the second kind $Q_{n}(x)$ satisfy the following orthogonality relation:

$$
\int_{E} Q_{n}(x) Q_{m}(x) \frac{1}{w(x)} d x=\delta_{n m} \tilde{h}_{n},
$$

where,

$$
\tilde{h}_{n}=\pi^{2} h_{n} .
$$

Proof. We wish to evaluate the following integral:

$$
I_{j}=\int_{E} \frac{Q_{n}(t)}{w(t)} t^{j} d t, \quad 0 \leq j<n-1 .
$$

We have

$$
\begin{aligned}
Q_{n}(z) & =P_{n}(z) \psi(z)-\int_{E} \frac{P_{n}(t)}{z-t} w(t) d t \\
& =P_{n}(z) \psi(z)-\frac{1}{z} \int_{E} \frac{P_{n}(t)}{1-\frac{t}{z}} w(t) d t \\
& =P_{n}(z) \psi(z)-\frac{1}{z} \int_{E} P_{n}(t) \sum_{j=0}^{\infty}\left(\frac{t}{z}\right)^{j} d t \\
& =P_{n}(z) \psi(z)-\left(\frac{h_{n}}{z^{n+1}}+O\left(\frac{1}{z^{n+2}}\right)\right)
\end{aligned}
$$


where the last line follows from the orthogonality of the $P_{n}(x)$. Therefore,

$$
\frac{Q_{n}(z)}{\psi(z)}=P_{n}(z)-\frac{1}{\psi(z)}\left(\frac{h_{n}}{z^{n+1}}+O\left(\frac{1}{z^{n+2}}\right)\right) .
$$

We rewrite $I_{j}$ as a contour integral using a closed contour $\Lambda$ [9] in the slit complex plane that encircles all of the branch points. By continuous deformation of the contour onto the intervals that make up $E$ we have

$$
\begin{aligned}
I_{j} & =\int_{E} \frac{Q_{n}(t)}{w(t)} t^{j} d t \\
& =\frac{\pi}{2 i} \int_{\Lambda} \frac{Q_{n}(z)}{\psi(z)} z^{j} d z .
\end{aligned}
$$

So $I_{j}$ can be written as

$$
I_{j}=\int_{\Lambda} P_{n}(z) z^{j} d z-\int_{\Lambda} \frac{\frac{h_{n}}{z^{n+1}}+O\left(\frac{1}{z^{n+2}}\right)}{\psi(z)} z^{j} d z
$$

The differential $P_{n}(z) z^{j} d z$ has no residue at infinity for any $j$. The differential $\frac{z^{j}}{z^{n+1} \psi(z)} d z$ has no residue at infinity for $j=0, \ldots, n-2$. Consequently, for these values of $j$, both integrals are zero, which proves the orthogonality of the $Q_{n}(z)$ with respect to $\frac{1}{w(z)}$. The integral $I_{n-1}$ is equal to the square of the weighted $L_{2}$ norm of $Q_{n}(z)$. In this case there is a residue at infinity. The residue is $-h_{n}$ and therefore

$$
\tilde{h}_{n}=\pi^{2} h_{n}
$$

Theorem 1.2. Let $\tilde{E}$ be the reflection of $E$ around the point $x=0$, namely

$$
\tilde{E}:=\left[-1,-\beta_{g}\right] \cup_{j=g-1}^{1}\left[-\alpha_{j+1},-\beta_{j}\right] \cup\left[-\alpha_{1}, 1\right] .
$$

Let $\left\{\tilde{P}_{n}(x)\right\}$ be the monic polynomials orthogonal on $\tilde{E}$ with respect to $\tilde{w}(x)$, where

$$
\tilde{w}(x)=\frac{1}{\pi} \sqrt{\frac{\prod_{j=1}^{g}\left(x+\beta_{j}\right)}{\left(1-x^{2}\right) \prod_{j=1}^{g}\left(x+\alpha_{j}\right)}} .
$$

Then we have for $n \geq g$

$$
\tilde{P}_{n}(x)=\frac{(-1)^{n+g}}{D_{n} \prod_{j=1}^{g}\left(x+\beta_{j}\right)}\left|\begin{array}{ccccc}
P_{n-g}\left(\beta_{1}\right) & \ldots & \ldots & \ldots & P_{n+g}\left(\beta_{1}\right) \\
\vdots & \vdots & \vdots & \vdots & \vdots \\
P_{n-g}\left(\beta_{g}\right) & \ldots & \ldots & \ldots & P_{n+g}\left(\beta_{g}\right) \\
Q_{n-g}\left(\alpha_{1}\right) & \ldots & \ldots & \ldots & Q_{n+g}\left(\alpha_{1}\right) \\
\vdots & \vdots & \vdots & \vdots & \vdots \\
Q_{n-g}\left(\alpha_{g}\right) & \ldots & \ldots & \ldots & Q_{n+g}\left(\alpha_{g}\right) \\
P_{n-g}(-x) & \ldots & P_{n}(-x) & \ldots & P_{n+g}(-x)
\end{array}\right| .
$$


If $n<g$, then

$$
\tilde{P}_{n}(x)=\frac{(-1)^{n+g}}{D_{n} \prod_{j=1}^{g}\left(x+\beta_{j}\right)}\left|\begin{array}{ccccccc}
0 & \ldots & \ldots & 0 & P_{0}\left(\beta_{1}\right) & \ldots & P_{n+g}\left(\beta_{1}\right) \\
\vdots & \vdots & \vdots & \vdots & \vdots & \vdots & \vdots \\
0 & \ldots & \ldots & 0 & P_{0}\left(\beta_{g}\right) & \ldots & P_{n+g}\left(\beta_{g}\right) \\
\alpha_{1}^{0} & \alpha_{1} & \ldots & \alpha_{1}^{g-n-1} & Q_{0}\left(\alpha_{1}\right) & \ldots & Q_{n+g}\left(\alpha_{1}\right) \\
\vdots & \vdots & \vdots & \vdots & \vdots & \vdots & \vdots \\
\alpha_{g}^{0} & \alpha_{g} & \ldots & \alpha_{g}^{g-n-1} & Q_{0}\left(\alpha_{g}\right) & \ldots & Q_{n+g}\left(\alpha_{g}\right) \\
0 & 0 & 0 & 0 & P_{0}(-x) & \ldots & P_{n+g}(-x)
\end{array}\right| .
$$

In both equations, $D_{n}$ is equal to the coefficient of $P_{n+g}(-x)$ appearing in each determinant.

Proof. Starting with the orthogonality relation of the $\tilde{P}_{n}(x)$ we make the change of variables $x \rightarrow-x$ :

$$
\begin{aligned}
\tilde{h}_{n} \delta_{n m} & =\int_{\tilde{E}} \tilde{P}_{n}(x) \tilde{P}_{m}(x) \sqrt{\frac{\prod_{j=1}^{g}\left(x+\beta_{j}\right)}{\left(1-x^{2}\right) \prod_{j=1}^{g}\left(x+\alpha_{j}\right)}} d x \\
& =\int_{E} \tilde{P}_{n}(-x) \tilde{P}_{m}(-x) \sqrt{\frac{\prod_{j=1}^{g}\left(x-\beta_{j}\right)}{\left(1-x^{2}\right) \prod_{j=1}^{g}\left(x-\alpha_{j}\right)}} d x \\
& =\int_{E} \tilde{P}_{n}(-x) \tilde{P}_{m}(-x) \sqrt{\frac{\prod_{j=1}^{g}\left(x-\alpha_{j}\right)}{\left(1-x^{2}\right) \prod_{j=1}^{g}\left(x-\beta_{j}\right)}}\left(\frac{\prod_{j=1}^{g}\left(x-\beta_{j}\right)}{\prod_{j=1}^{g}\left(x-\alpha_{j}\right)}\right) d x .
\end{aligned}
$$

So the $\tilde{P}_{n}(-x)$ are orthogonal with respect to a weight function that is $w(x)$ multiplied by a rational function. In general if we know the polynomials orthogonal with respect to a weight $\mu(x)$ and we wish to find the polynomials orthogonal with respect to $R(x) \mu(x)$ where $R(x)$ is a rational function that is positive on the interval of orthogonality, we may invoke a theorem of Uvarov that gives these polynomials in terms of the original ones. The theorem was first published in 18 and also appears in [19. In our case the rational function has zeros and poles at the end points of the subintervals that make up $E$. However, a brief examination of the proof will show that the theorem is still applicable in this case. The result follows directly from the theorem.

In section 2 we wish to study the following polynomials:

$$
S_{g}(x ; n):=P_{n}^{2}(x) \prod_{j=1}^{g}\left(x-\alpha_{j}\right)-Q_{n}^{2}(x)\left(x^{2}-1\right) \prod_{j=1}^{g}\left(x-\beta_{j}\right)
$$

and

$$
G_{g+1}(x ; n):=P_{n}(x) P_{n-1}(x) \prod_{j=1}^{g}\left(x-\alpha_{j}\right)-Q_{n}(x) Q_{n-1}(x)\left(x^{2}-1\right) \prod_{j=1}^{g}\left(x-\beta_{j}\right) .
$$

These functions are fundamental in the theory of the generalizations of Chebyshev polynomials considered here.

Theorem 1.3. The functions $S_{g}(x ; n)$ and $G_{g+1}(x ; n)$ are polynomials of degree $g$ and $g+1$ respectively. 
Proof. From (1.3) we know that

$$
Q_{n}(z)=P_{n}(z) \psi(z)-\int_{E} \frac{P_{n}(t)}{z-t} w(t) d t .
$$

Expanding the integrand in powers of $t$ we have

$$
\frac{1}{z-t}=\frac{1}{z}+\frac{t}{z^{2}}+\frac{t^{2}}{z^{3}}+\ldots
$$

Therefore, as $z \rightarrow \infty^{+}$, we have

$$
P_{n}(z) \psi(z)-Q_{n}(z)=O\left(\frac{1}{z^{n+1}}\right),
$$

and similarly,

$$
P_{n}(z) \psi(z)+Q_{n}(z)=O\left(z^{n-1}\right) .
$$

Multiplying the two together gives

$$
P_{n}^{2}(z) \psi^{2}(z)-Q_{n}^{2}(z)=O\left(\frac{1}{z^{2}}\right) .
$$

Therefore,

$$
\prod_{j=1}^{g}\left(z-\alpha_{j}\right) P_{n}^{2}(z)-\left(z^{2}-1\right) \prod_{j=1}^{g}\left(z-\beta_{j}\right) Q_{n}^{2}(z)=O\left(z^{g}\right) .
$$

Since the left hand side is a polynomial it follows that it must be of degree $g$. For (1.14) we form the following product:

$$
\begin{aligned}
\left(P_{n}(z) \psi(z)-\right. & \left.Q_{n}(z)\right)\left(P_{n-1}(z) \psi(z)+Q_{n-1}(z)\right) \\
& =P_{n}(z) P_{n-1}(z) \psi(z)-Q_{n}(z) Q_{n-1}(z)-\psi(z) h_{n-1}
\end{aligned}
$$

where we have used

$$
P_{n-1}(z) Q_{n}(z)-P_{n}(z) Q_{n-1}(z)=h_{n-1},
$$

which is the Wronskian. As a result,

$$
\prod_{j=1}^{g}\left(z-\alpha_{j}\right) P_{n}(z) P_{n-1}(z)-\left(z^{2}-1\right) \prod_{j=1}^{g}\left(z-\beta_{j}\right) Q_{n}(z) Q_{n-1}(z)=O\left(z^{g+1}\right) .
$$

From (1.13) and (1.14) we have

$$
\mathcal{E}_{n}\left(\mathfrak{p}_{x}\right) \tilde{\mathcal{E}}_{n}\left(\mathfrak{p}_{x}\right)=\frac{S_{g}(x ; n)}{\prod_{j=1}^{g}\left(x-\alpha_{j}\right)} .
$$

Also,

$$
\begin{aligned}
& \mathcal{E}_{n}\left(\mathfrak{p}_{x}\right) \tilde{\mathcal{E}}_{n-1}\left(\mathfrak{p}_{x}\right)=\frac{G_{g+1}(x ; n)+h_{n-1} y(x)}{\prod_{j=1}^{g}\left(x-\alpha_{j}\right)}, \\
& \tilde{\mathcal{E}}_{n}\left(\mathfrak{p}_{x}\right) \mathcal{E}_{n-1}\left(\mathfrak{p}_{x}\right)=\frac{G_{g+1}(x ; n)-h_{n-1} y(x)}{\prod_{j=1}^{g}\left(x-\alpha_{j}\right)} .
\end{aligned}
$$

An alternative representation for the polynomials of the first and second kind was also given in 9], resulting from a coupled system of differential equations

$$
P_{n}(z):=\rho_{n}(z) \cos \Psi_{n}(z)
$$


and

$$
Q_{n}(z):=i \psi(z) \rho_{n}(z) \sin \Psi_{n}(z)
$$

where

$$
\rho_{n}(z)=\sqrt{\frac{S_{g}(z ; n)}{\prod_{j=1}^{g}\left(z-\alpha_{j}\right)}}
$$

and

$$
\Psi_{n}(z)=i \int_{1}^{z} \frac{\left(\sum_{j=0}^{g-1} c_{j}(n) t^{j}-n t^{g}+\frac{1}{2} \sum_{j=1}^{g} \frac{y_{j}(n)}{t-\gamma_{j}(n)}\right)}{y} d t
$$

where the branch of the square root is chosen in such a way that $\rho_{n}(z) \rightarrow \sqrt{2 h_{n}}$, as $\Re z \rightarrow \infty$ and $2 h_{n}$ is the leading coefficient of $S_{g}(z ; n)$, to be shown later. Here the path of integration is from 1 to an arbitrary complex point $z$ with the property $\Im z>0$, and the path of integration is entirely in the upper half plane. Expressions for the real values are then obtained by the analytical continuation of those above allowing $z \rightarrow x \in \mathbb{R}$.

In these expressions, the $\gamma_{j}(n)$ are the zeroes of $S_{g}(x, n)$, and $y_{i}=\left.y\left(\mathfrak{p}_{x}\right)\right|_{\mathfrak{p}_{x}=\gamma_{j}(n)}$. The $c_{j}(n)$ are the first $g+1$ expansion coefficients of $\frac{d}{d x} \ln \mathcal{E}_{n}\left(\mathfrak{p}_{x}\right)$ in terms of the following basis:

$$
\left\{\frac{1}{y}, \frac{x}{y}, \ldots, \frac{x^{g}}{y}, \frac{1}{x-\alpha_{1}}, \ldots, \frac{1}{x-\alpha_{g}}, \frac{y+y_{1}}{y\left(x-\gamma_{1}(n)\right)}, \ldots, \frac{y+y_{g}}{y\left(x-\gamma_{g}(n)\right)}\right\} .
$$

This is the basis for meromorphic functions on the Riemann surface defined by $y(x)$ whose zeroes must include simple zeroes at $\pm \infty$ and whose poles must be from amongst the set $\mathfrak{p}_{1}, \mathfrak{p}_{-1}, \mathfrak{p}_{\alpha_{j}}, \mathfrak{p}_{\beta_{j}}, \mathfrak{p}_{\gamma_{j}}, j=1, \ldots, g$, where the poles at the $\alpha$ points can be at most double poles and the other poles must be simple poles.

\section{Evaluating the polynomials $S_{g}(x ; n)$ And $G_{g+1}(x ; n)$}

In this section we give an algorithm for determining the coefficients of $S_{g}(x ; n)$ and $G_{g+1}(x ; n)$ in terms of the recurrence coefficients and the branch points. Let

$$
S_{g}(x ; n)=\sum_{j=0}^{g} \eta_{j}(n) x^{j}
$$

and

$$
G_{g+1}(x ; n)=\sum_{j=0}^{g+1} \xi_{j}(n) x^{j}
$$

Then we have,

$$
\begin{gathered}
\psi(z) P_{n}^{2}(z)-\frac{Q_{n}^{2}(z)}{\psi(z)}=\frac{\eta_{g}(n) z^{g}+\ldots+\eta_{0}(n)}{y(z)}, \\
\psi(z) P_{n}(z) P_{n-1}(z)-\frac{Q_{n}(z) Q_{n-1}(z)}{\psi(z)}=\frac{\xi_{g+1}(n) z^{g+1}+\ldots+\xi_{0}(n)}{y(z)} .
\end{gathered}
$$

To find the $\eta_{j}(n)$ coefficients we integrate both sides of (2.1) along the contour $\Lambda$. Note that,

$$
\psi(z)=\frac{\pi}{i} W(z),
$$


where

$$
W(z)=\frac{i}{\pi} \sqrt{\frac{\prod_{j=1}^{g}\left(z-\alpha_{j}\right)}{\left(z^{2}-1\right) \prod_{j=1}^{g}\left(z-\beta_{j}\right)}}
$$

and for $t \in E$,

$$
\lim _{\epsilon \rightarrow 0+} W(t+i \epsilon)=w(t):=\frac{1}{\pi} \sqrt{\frac{\prod_{j=1}^{g}\left(t-\alpha_{j}\right)}{\left(1-t^{2}\right) \prod_{j=1}^{g}\left(t-\beta_{j}\right)}} .
$$

We obtain the following:

$$
\frac{\pi}{i} \int_{\Lambda} W(z) P_{n}^{2}(z) d z-\frac{i}{\pi} \int_{\Lambda} \frac{Q_{n}^{2}(z)}{W(z)} d z=\sum_{j=0}^{g} \eta_{j}(n) I_{j}
$$

where

$$
I_{j}=\int_{\Lambda} \frac{z^{j}}{y(z)} d z
$$

The $I_{j}$ can be evaluated by evaluating the residue at infinity, in which case,

$$
I_{j}=0, \quad j<g,
$$

and

$$
I_{g+k}=\int_{\Lambda} \frac{z^{g+k}}{\left[\left(z^{2}-1\right) \prod_{j=1}^{g}\left(z-\alpha_{j}\right)\left(z-\beta_{j}\right)\right]^{1 / 2}} d z
$$

which under the substitution $z=1 / Z$ becomes

$$
I_{g+k}=-\int_{\Omega} \frac{d Z}{Z^{k+1}\left[\left(1-Z^{2}\right) \prod_{j=1}^{g}\left(1-\alpha_{j} Z\right)\left(1-\beta_{j} Z\right)\right]^{1 / 2}} .
$$

So in general the integrand of $I_{g+k}$ has a pole of order $k+1$ at infinity. Hence if

$$
f(Z)=\left[\left(1-Z^{2}\right) \prod_{j=1}^{g}\left(1-\alpha_{j} Z\right)\left(1-\beta_{j} Z\right)\right]^{-1 / 2},
$$

then

$$
I_{g+k}=-2 \pi i \frac{f^{(k)}(0)}{k !}
$$

where $f^{(k)}(Z)$ denotes the $k^{\text {th }}$ derivative of $f$. Deforming $\Lambda$ onto $E(2.4)$ becomes

$$
4 h_{n}=\frac{i}{\pi} \eta_{g}(n) I_{g}
$$

From (2.5) we have,

$$
I_{g}=-2 \pi i
$$

and therefore,

$$
\eta_{g}(n)=2 h_{n}
$$


To find the remaining $\eta_{j}(n)$ coefficients we multiply (2.1) by successive powers of $z$ and perform the same integration. This leaves us with the following system of equations that we can use to solve for the $\eta_{g}(n)$ :

$$
\begin{aligned}
r_{0}(n) & =I_{g} \eta_{g}(n), \\
r_{1}(n) & =I_{g+1} \eta_{g}(n)+I_{g} \eta_{g-1}(n), \\
r_{2}(n) & =I_{g+2} \eta_{g}(n)+I_{g+1} \eta_{g-1}(n)+I_{g} \eta_{g-2}(n) \\
& \vdots \\
r_{g}(n) & =\sum_{j=0}^{g} I_{g+j} \eta_{j} .
\end{aligned}
$$

Here,

$$
r_{k}(n)=\frac{4 \pi}{i} \int_{E} z^{k} P_{n}^{2}(z) w(z) d z .
$$

These constants are obtained by iterating the three term recurrence relation $k$ times in (2.6). The recurrence relation can also be expressed in matrix form by

$$
L p=z p,
$$

where

$$
L=\left(\begin{array}{ccccccc}
b_{1} & 1 & 0 & 0 & 0 & 0 & 0 \\
a_{1} & b_{2} & 1 & 0 & 0 & 0 & 0 \\
0 & a_{2} & b_{3} & 1 & 0 & 0 & 0 \\
0 & 0 & a_{3} & b_{4} & 1 & 0 & 0 \\
0 & 0 & 0 & a_{4} & b_{5} & 1 & 0 \\
0 & 0 & 0 & 0 & \ddots & \ddots & \ddots \\
0 & 0 & 0 & 0 & 0 & \ddots & \ddots
\end{array}\right) \quad p=\left(\begin{array}{c}
P_{0}(z) \\
P_{1}(z) \\
P_{2}(z) \\
P_{3}(z) \\
\vdots \\
\vdots \\
\vdots
\end{array}\right)
$$

In this case,

$$
\begin{aligned}
z^{k} p & =L^{k} p \\
z P_{n}(z) & =\sum_{m=0}^{\infty} L_{n m} p_{m}, \\
z^{k} P_{n}(z) & =\sum_{m=0}^{\infty}\left[L^{k}\right]_{n m} P_{m}(z) .
\end{aligned}
$$

From the expression for $r_{k}(n)$ in (2.6) it is clear that

$$
r_{k}(n)=\frac{4 \pi h_{n}}{i}\left[L^{k}\right]_{n-1, n}
$$

To find the $\xi_{j}(n)$ we use the same method. We know from the proof of theorem (1.3) that

$$
\xi_{g+1}(n)=h_{n-1}
$$


Multiplying (2.2) by successive powers of $z$ and integrating we have the following system of equations:

$$
\begin{aligned}
0 & =I_{g+1} \xi_{g+1}(n)+I_{g} \xi_{g}(n), \\
s_{1}(n) & =I_{g+2} \xi_{g+1}(n)+I_{g+1} \xi_{g}(n)+I_{g} \xi_{g-1}(n), \\
s_{2}(n) & =I_{g+3} \xi_{g+1}(n)+I_{g+2} \xi_{g}(n)+I_{g+1} \xi_{g-1}(n)+I_{g} \xi_{g-2}(n) \\
& \vdots \\
s_{g}(n) & =\sum_{j=0}^{g+1} I_{g+j} \xi_{j}(n) .
\end{aligned}
$$

Here,

$$
s_{k}(n)=\frac{4 \pi}{i} \int_{E} z^{k} P_{n}(z) P_{n-1}(z) w(z) d z .
$$

In terms of the Jacobi matrix,

$$
s_{k}(n)=\frac{4 \pi h_{n}}{i}\left[L^{k}\right]_{n n} .
$$

For $g=1$ we have,

$$
\begin{aligned}
S_{1}(x, n) & =2 h_{n}\left(x+b_{n+1}-\frac{(\alpha+\beta)}{2}\right), \quad n \geq 1, \\
G_{2}(x, n) & =h_{n-1}\left(x^{2}-\frac{\alpha+\beta}{2} x+2 a_{n}-\frac{1}{8}(\alpha-\beta)^{2}-\frac{1}{2}\right), \quad n \geq 2, \\
S_{1}(x, 0) & =(x-\alpha), \\
G_{2}(x, 1) & =\left(x-b_{1}\right)(x-\alpha) .
\end{aligned}
$$

The auxiliary polynomials for $g=2$ can be found in Appendix B.

Non-linear difference equations. We can use the polynomials studied in this section to derive a pair of non-linear difference equations for each genus that are satisfied by the recurrence coefficients $\left\{a_{n}\right\}$ and $\left\{b_{n}\right\}$. Using the three term recurrence relation,

$$
\begin{aligned}
& \frac{S_{g}(z ; n)}{\prod_{j=1}^{g}\left(z-\alpha_{j}\right)}=\mathcal{E}_{n}\left(\mathfrak{p}_{z}\right) \tilde{\mathcal{E}}_{n}\left(\mathfrak{p}_{z}\right) \\
& \quad=\left(z-b_{n}\right)^{2} \mathcal{E}_{n-1}\left(\mathfrak{p}_{z}\right) \tilde{\mathcal{E}}_{n-1}\left(\mathfrak{p}_{z}\right) \\
& \quad-a_{n-1}\left(z-b_{n}\right)\left(\mathcal{E}_{n-2}\left(\mathfrak{p}_{z}\right) \tilde{\mathcal{E}}_{n-1}\left(\mathfrak{p}_{z}\right)+\tilde{\mathcal{E}}_{n-2}\left(\mathfrak{p}_{z}\right) \mathcal{E}_{n-1}\left(\mathfrak{p}_{z}\right)\right) \\
& \quad+a_{n-1}^{2} \mathcal{E}_{n-2}\left(\mathfrak{p}_{z}\right) \tilde{\mathcal{E}}_{n-2}\left(\mathfrak{p}_{z}\right) \\
& \quad=\frac{\left(z-b_{n}\right)^{2} S_{g}(z ; n-1)+a_{n-2}^{2} S_{g}(z ; n-2)-2 a_{n-1}\left(z-b_{n}\right) G_{g+1}(z ; n-1)}{\prod_{j=1}^{g}\left(z-\alpha_{j}\right)} .
\end{aligned}
$$

Evaluating both sides at $z=b_{n}$ gives

$$
S_{g}\left(b_{n} ; n\right)=a_{n-1}^{2} S_{g}\left(b_{n}, n-2\right) .
$$

If we write this out fully for $g=1$ we have

$$
a_{n}\left(b_{n+1}+b_{n}-\frac{\alpha+\beta}{2}\right)=a_{n-1}\left(b_{n}+b_{n-1}-\frac{\alpha+\beta}{2}\right) .
$$


This is the first difference relation for the recurrence coefficients. To get the second we note that

$$
\begin{aligned}
\frac{G_{g+1}(z ; n)}{\prod_{j=1}^{g}\left(z-\alpha_{j}\right)}= & \frac{1}{2}\left(\mathcal{E}_{n}\left(\mathfrak{p}_{z}\right) \tilde{\mathcal{E}}_{n-1}\left(\mathfrak{p}_{z}\right)+\tilde{\mathcal{E}}_{n}\left(\mathfrak{p}_{z}\right) \mathcal{E}_{n-1}\left(\mathfrak{p}_{z}\right)\right) \\
= & \frac{1}{2}\left(\left(\left(z-b_{n}\right) \mathcal{E}_{n-1}\left(\mathfrak{p}_{z}\right)-a_{n-1} \mathcal{E}_{n-2}\left(\mathfrak{p}_{z}\right)\right) \tilde{\mathcal{E}}_{n-1}\left(\mathfrak{p}_{z}\right)\right. \\
& \left.+\left(\left(z-b_{n}\right) \tilde{\mathcal{E}}_{n-1}\left(\mathfrak{p}_{z}\right)-a_{n-1} \tilde{\mathcal{E}}_{n-2}\left(\mathfrak{p}_{z}\right)\right) \mathcal{E}_{n-1}\left(\mathfrak{p}_{z}\right)\right) \\
= & \left(z-b_{n}\right) \mathcal{E}_{n-1}\left(\mathfrak{p}_{z}\right) \tilde{\mathcal{E}}_{n-1}\left(\mathfrak{p}_{z}\right) \\
& -\frac{a_{n-1}}{2}\left(\mathcal{E}_{n-2}\left(\mathfrak{p}_{z}\right) \tilde{\mathcal{E}}_{n-1}\left(\mathfrak{p}_{z}\right)+\tilde{\mathcal{E}}_{n-2}\left(\mathfrak{p}_{z}\right) \mathcal{E}_{n-1}\left(\mathfrak{p}_{z}\right)\right) \\
= & \frac{\left(z-b_{n}\right) S_{g}(z ; n-1)-a_{n-1} G_{g+1}(z ; n-1)}{\prod_{j=1}^{g}\left(z-\alpha_{j}\right)} .
\end{aligned}
$$

If we evaluate this at $z=b_{n}$ we get

$$
G_{g+1}\left(b_{n} ; n\right)=-a_{n-1} G_{g+1}\left(b_{n} ; n-1\right) .
$$

Writing this out for genus 1 ,

$$
a_{n}+a_{n-1}=\frac{1}{2}+\frac{(\beta-\alpha)^{2}}{8}+\frac{\alpha+\beta}{2} b_{n}-b_{n}^{2} .
$$

This is the second difference equation for the recurrence coefficients. Both equations are valid for $n \geq 3$.

\section{Product Representation for $P_{n}(x)$ And $Q_{n}(x)$}

In this section we use the polynomials derived in section 2 to derive a representation for the generalized Chebyshev polynomials where the coefficients of the powers of $x$ are given in terms of the branch points and the recurrence coefficients. From (1.5) we have

$$
P_{n}(x)=\frac{1}{2}\left(\mathcal{E}_{n}\left(\mathfrak{p}_{x}\right)+\tilde{\mathcal{E}}_{n}\left(\mathfrak{p}_{x}\right)\right) .
$$

We define the following functions:

$$
\begin{aligned}
f_{+}(x ; n) & :=\frac{\mathcal{E}_{n}\left(\mathfrak{p}_{x}\right)}{\mathcal{E}_{n-1}\left(\mathfrak{p}_{x}\right)}, \\
f_{-}(x ; n) & :=\frac{\tilde{\mathcal{E}}_{n}\left(\mathfrak{p}_{x}\right)}{\tilde{\mathcal{E}}_{n-1}\left(\mathfrak{p}_{x}\right)} .
\end{aligned}
$$

Using (1.13) and (1.14) we can write

$$
f_{+}(x ; n)=\frac{\mathcal{E}_{n}\left(\mathfrak{p}_{x}\right) \tilde{\mathcal{E}}_{n-1}\left(\mathfrak{p}_{x}\right)}{\mathcal{E}_{n-1}\left(\mathfrak{p}_{x}\right) \tilde{\mathcal{E}}_{n-1}\left(\mathfrak{p}_{x}\right)}=\frac{G_{g+1}(x ; n)+h_{n-1} y(x)}{S_{g}(x ; n-1)} .
$$

Similarly,

$$
f_{-}(x ; n)=\frac{\tilde{\mathcal{E}}_{n}\left(\mathfrak{p}_{x}\right) \mathcal{E}_{n-1}\left(\mathfrak{p}_{x}\right)}{\tilde{\mathcal{E}}_{n-1}\left(\mathfrak{p}_{x}\right) \mathcal{E}_{n-1}\left(\mathfrak{p}_{x}\right)}=\frac{G_{g+1}(x ; n)-h_{n-1} y(x)}{S_{g}(x ; n-1)} .
$$

Since, $\mathcal{E}_{0}\left(\mathfrak{p}_{x}\right)=1$, we have, for any $x \in \mathbb{C}$

$$
P_{n}(x)=\frac{1}{2}\left(\prod_{j=1}^{n} f_{+}(x, j)+\prod_{j=1}^{n} f_{-}(x, j)\right) .
$$


Similarly,

$$
Q_{n}(x)=\frac{\psi(x)}{2}\left(\prod_{j=1}^{n} f_{+}(x, j)-\prod_{j=1}^{n} f_{-}(x, j)\right) .
$$

(3.3) and (3.4) are valid for any genus $g$ and require only knowledge of the branch points and the recurrence coefficients. They do not require any knowledge of the $\gamma$ points. If we look at the genus 1 case we find that

$$
f_{ \pm}(x ; n)=\frac{x^{2}-\frac{\alpha+\beta}{2} x+2 a_{n}-\frac{1}{8}(\alpha-\beta)^{2}-\frac{1}{2} \pm \sqrt{\left(x^{2}-1\right)(x-\alpha)(x-\beta)}}{2\left(x+b_{n+1}-\frac{\alpha+\beta}{2}\right)} .
$$

If we take the limit as $\alpha \rightarrow \beta$ we see that

$$
\begin{gathered}
S_{1}(x, n) \rightarrow 2 h_{n}(x-\alpha), \quad n \geq 1, \\
G_{2}(x, n) \rightarrow h_{n-1} x(x-\alpha),
\end{gathered}
$$

and,

Note that

$$
f_{ \pm}(x, n) \rightarrow \frac{1}{2}\left(x \pm \sqrt{x^{2}-1}\right)=\frac{1}{2} e^{ \pm i \theta}, \quad x=\cos \theta, \quad n \geq 2 .
$$

$$
f_{ \pm}(x, 1) \rightarrow e^{ \pm i \theta}
$$

Thus we can see that (3.3) is a natural generalization of the following formula for the classical Chebyshev polynomials:

$$
T_{n}(x)=\frac{\left(x+\sqrt{x^{2}-1}\right)^{n}+\left(x-\sqrt{x^{2}-1}\right)^{n}}{2^{n}} .
$$

In general, (3.3) and (3.4) are very effective representations of the polynomials for the purpose of extracting the polynomial coefficients. The appearance of the theta function of the Riemann surface is still apparent in the expression for the recurrence coefficients. One may also wish to study certain special cases of the Akhiezer polynomials when the recurrence coefficients become algebraic functions of the branch points $\alpha_{i}$ and $\beta_{i}$. In this case (3.3) is particularly useful, as one could insert directly the value of the recurrence coefficients. In the next section we will encounter some of these special cases. The following formulas follow naturally from (3.3):

$$
P_{n}(x)=\frac{G_{g+1}(x, n)}{S_{g}(x, n-1)} P_{n-1}(x)+\frac{h_{n-1}\left(x^{2}-1\right) \prod_{j=1}^{g}\left(x-\beta_{j}\right)}{S_{g}(x, n-1)} Q_{n-1}(x),
$$

and the corresponding formula for the $Q_{n}$ is

$$
Q_{n}(x)=\frac{G_{g+1}(x, n)}{S_{g}(x, n-1)} Q_{n-1}(x)+\frac{h_{n-1} \prod_{j=1}^{g}\left(x-\alpha_{j}\right)}{S_{g}(x, n-1)} P_{n-1}(x) .
$$

Letting $\alpha_{i} \rightarrow \beta_{i}$ in (3.5) we obtain as expected the analogous formula for the Chebyshev case:

$$
2 T_{n}(x)=x T_{n-1}(x)+\left(x^{2}-1\right) U_{n-1}(x) .
$$

Here $T_{n}(x)$ and $U_{n}(x)$ are monic Chebyshev polynomials of the first and second kind respectively. Now we show how the product representations of (3.3) and (3.4) can be used to re-express the differential relations derived in [9], where $P_{n}(x)$ and the $Q_{n}(x)$ were shown to satisfy

$$
P_{n}^{\prime}(x)=f_{1}(x ; n) P_{n}(x)+f_{2}(x ; n) Q_{n}(x),
$$


and

$$
Q_{n}^{\prime}(x)=f_{3}(x ; n) P_{n}(x)+f_{4}(x ; n) Q_{n}(x),
$$

where

$$
\begin{gathered}
f_{1}(x ; n)=\frac{1}{2} \sum_{j=1}^{g}\left(\frac{1}{x-\gamma_{j}}-\frac{1}{x-\alpha_{j}}\right) \\
f_{2}(x ; n)=\frac{n x^{g}-\sum_{j=0}^{g-1} c_{j} x^{j}-\frac{1}{2} \sum_{j=1}^{g} \frac{y_{j}}{x-\gamma_{j}}}{\prod_{j=1}^{g}\left(x-\alpha_{j}\right)}
\end{gathered}
$$

and

$$
\begin{gathered}
f_{3}(x ; n)=\psi^{2}(x) f_{2}(x ; n), \\
f_{4}(x ; n)=\frac{\psi^{\prime}(x)}{\psi(x)}+f_{1}(x ; n) .
\end{gathered}
$$

The definitions of all the constants appearing in the above formulas can be found in section 1. From [9,

$$
f_{1}(x ; n)=\frac{\rho_{n}^{\prime}(x)}{\rho_{n}(x)}
$$

and

$$
f_{2}(x ; n)=i \frac{\Psi_{n}^{\prime}(x)}{\psi(x)} .
$$

Using (3.1) we can express $f_{1}(x ; n)$ and $f_{2}(x ; n)$ in more explicit terms. Recall that

$$
\rho_{n}(x)=\sqrt{\frac{S_{g}(x, n)}{\prod_{j=1}^{g}\left(x-\alpha_{j}\right)}} .
$$

Therefore, we can write

$$
f_{1}(x ; n)=\frac{\rho_{n}^{\prime}(x)}{\rho_{n}(x)}=\frac{1}{2}\left(\frac{S_{g}^{\prime}(x, n)}{S_{g}(x, n)}-\sum_{j=1}^{g} \frac{1}{x-\alpha_{j}}\right) .
$$

This is simply a restatement of the earlier definition if we recall that the $\gamma_{i}(n)$ are the zeroes of $S_{g}(x, n)$. In order to re-express $f_{2}(x ; n)$, we observe that

$$
\prod_{j=1}^{n} f_{ \pm}(x, j)=\rho_{n}(x) e^{ \pm i \Psi_{n}(x)} .
$$

Therefore,

$$
\prod_{j=1}^{n} \frac{f_{+}(x, j)}{f_{-}(x, j)}=e^{2 i \Psi_{n}(x)} \quad \text { or } \quad \ln \prod_{j=1}^{n} \frac{f_{+}(x, j)}{f_{-}(x, j)}=2 i \Psi_{n}(x) \quad(\bmod 2 \pi) .
$$

From the definitions of $f_{ \pm}(x, j)$ we have

$$
2 i \Psi_{n}(x)=\sum_{j=1}^{n} \ln \left(\frac{G_{g+1}(x ; j)+h_{j-1} y(x)}{G_{g+1}(x ; j)-h_{j-1} y(x)}\right) \quad(\bmod 2 \pi) .
$$

Hence,

$$
i \Psi_{n}^{\prime}(x)=\sum_{j=1}^{n}\left[\frac{y^{\prime}(x) G_{g+1}(x ; j)-y(x) G_{g+1}^{\prime}(x ; j)}{S_{g}(x ; j) S_{g}(x ; j-1)}\right] h_{j-1} .
$$


Substituting the above into (3.9) gives us the differential relations (3.7) and (3.8) completely in terms of the recurrence coefficients and the branch points. Note that, generally when $\gamma_{l}(k) \in\left(\alpha_{l}, \beta_{l}\right), \quad k \geq 1$, we can evaluate $\Psi_{n}\left(\alpha_{l}\right)$ by taking the limit $x \rightarrow \alpha_{l}$ in (3.11). From (1.13) and (1.14) we see that for $x=\alpha_{l}$ the only non-zero term appearing in the sum in (3.11) is the $j=1$ term. For $j>1, G_{g+1}\left(\alpha_{l} ; j\right)$ cannot be zero since this would imply, from (1.13) and (1.14), that $\gamma_{l}(j)=\alpha_{l}$. We have

$$
\lim _{x \rightarrow \alpha_{l}} 2 i \Psi_{n}(x)=\lim _{x \rightarrow \alpha_{l}} \ln \left(\frac{G_{g+1}(x ; 1)+y(x)}{G_{g+1}(x ; 1)-y(x)}\right) \quad(\bmod 2 \pi) .
$$

We know that

$$
G_{g+1}(x ; 1)=\left(x-b_{1}\right) \prod_{j=1}^{g}\left(x-\alpha_{j}\right)
$$

and therefore,

$$
\lim _{x \rightarrow \alpha_{l}} \Psi_{n}(x)=\frac{\pi}{2} \quad(\bmod \pi) .
$$

Discriminants. In this section we derive an expression for the discriminant when $g=1$. Once we have knowledge of the differential relations satisfied by a sequence of polynomials one can in general use this information to say something about the discriminant. The discriminant is useful when we consider certain electrostatic problems regarding the zeroes of the polynomials. See [16] and [13] for more details. The expressions we derive in this section are restricted to the cases when the polynomials do not have zeroes at the branch points. If we write

$$
P_{n}(x)=\prod_{j=1}^{n}\left(x-x_{j, n}\right),
$$

then the discriminant is defined to be the following:

$$
D\left[P_{n}(x)\right]:=\prod_{1 \leq i<j \leq n}\left(x_{i, n}-x_{j, n}\right)^{2} .
$$

Since

$$
P_{n}^{\prime}(x)=\sum_{j=1}^{n} \frac{P_{n}(x)}{\left(x-x_{j, n}\right)}
$$

it is easily verified that

$$
(-1)^{\frac{n(n-1)}{2}} \prod_{j=1}^{n} P_{n}^{\prime}\left(x_{j, n}\right)=D\left[P_{n}(x)\right] .
$$

From (3.7), assuming that $P_{n}(x)$ has no zeroes at the branch points, we have

$$
P_{n}^{\prime}\left(x_{j, n}\right)=f_{2}\left(x_{j, n} ; n\right) Q_{n}\left(x_{j, n}\right) .
$$

To evaluate $Q_{n}\left(x_{j, n}\right)$, recall that

$$
P_{n}(x)=\rho_{n}(x) \cos \Psi_{n}(x),
$$

and

$$
Q_{n}(x)=i \psi(x) \rho_{n}(x) \sin \Psi_{n}(x) .
$$

Since we have

$$
\frac{1}{i \psi(x)} \frac{Q_{n}(x)}{P_{n}(x)}=\tan \Psi_{n}(x),
$$


it must be that

$$
\tan \Psi_{n}\left(x_{j, n}\right)= \pm \infty
$$

and therefore if $x_{j, n}$ is a zero of $P_{n}(x)$, then we do have

$$
\cos \Psi_{n}\left(x_{j, n}\right)=0 .
$$

Hence,

$$
\sin \Psi_{n}\left(x_{j, n}\right)= \pm 1
$$

Therefore, it is always true that

$$
\left|Q_{n}\left(x_{j, n}\right)\right|=\left|i \psi\left(x_{j, n}\right) \rho_{n}\left(x_{j, n}\right)\right| .
$$

The sign depends on the value of $n$ and $j$. To show this dependence, we note that the quantity

$$
i \psi\left(x_{j, n}\right) \rho_{n}\left(x_{j, n}\right)=i \sqrt{\frac{2 h_{n}}{x_{j, n}^{2}-1} \prod_{l=1}^{g} \frac{x_{j, n}-\gamma_{l}(n)}{x_{j, n}-\beta_{l}}}
$$

is positive. Since theorem (7.1) in [9] states that any zero of $P_{n}(x)$ on the interval $\left(\alpha_{l}, \beta_{l}\right)$ must lie in $\left(\alpha_{l}, \gamma_{l}\right)$ and since the zeroes of $P_{n}(x)$ and $Q_{n}(x)$ interlace, we must have

$$
\sin \Psi_{2 n}\left(x_{j, 2 n}\right)=(-1)^{j}
$$

and

$$
\sin \Psi_{2 n-1}\left(x_{j, 2 n-1}\right)=(-1)^{j+1}
$$

Therefore,

and

$$
Q_{n}\left(x_{j, n}\right)=(-1)^{n+j} i \psi\left(x_{j, n}\right) \rho_{n}\left(x_{j, n}\right)
$$

$$
\begin{aligned}
D\left[P_{n}(x)\right] & =(-1)^{\frac{n(n-1)}{2}} \prod_{j=1}^{n} P_{n}^{\prime}\left(x_{j, n}\right) \\
& =(-1)^{\frac{n(n-1)}{2}} \prod_{j=1}^{n}(-1)^{n+j+\frac{1}{2}} \sqrt{2 h_{n}} \sqrt{\frac{\prod_{i=1}^{g}\left(x_{j, n}-\gamma_{i}(n)\right)}{\left(x_{j, n}^{2}-1\right) \prod_{i=1}^{g}\left(x_{j, n}-\beta_{i}\right)}} f_{2}\left(x_{j, n}\right) \\
3.14) & =(-1)^{\frac{n}{2}}\left(2 h_{n}\right)^{\frac{n}{2}} \sqrt{\frac{\prod_{j=1}^{g} P_{n}\left(\gamma_{j}(n)\right)}{P_{n}(1) P_{n}(-1) \prod_{j=1}^{g} P_{n}\left(\beta_{j}\right)}} \prod_{j=1}^{n} f_{2}\left(x_{j, n}\right) .
\end{aligned}
$$

Now in this most general form we cannot yet evaluate the discriminant for general $g$ since we do not know how to evaluate the product of the $f_{2}\left(x_{j, n}\right)$. However, examining the form of $f_{2}(x ; n)$ we see that it is a rational function. In certain special cases we may know enough about this function to factor it in which case we can express the unknown quantity in (3.14) as $P_{n}(x)$ evaluated at the zeroes and poles of $f_{2}(x ; n)$. For $g=1$ we can always do this, in which case,

$$
D\left[P_{n}(x)\right]=(-1)^{\frac{n}{2}}\left(2 h_{n}\right)^{\frac{n}{2}} \sqrt{\frac{P_{n}(\gamma(n))}{P_{n}(1) P_{n}(-1) P_{n}(\beta)}} \prod_{j=1}^{n} f_{2}\left(x_{j, n}\right) .
$$

Now

where

$$
f_{2}(x ; n)=\frac{n\left(x-r_{+}(n)\right)\left(x-r_{-}(n)\right)}{(x-\gamma(n))(x-\alpha)}
$$

$$
r_{ \pm}(n)=\frac{\left(c_{0} / n+\gamma\right) \pm \sqrt{\left(c_{0} / n-\gamma\right)^{2}+2 y_{1} / n}}{2}
$$


Therefore, we have for the square of the discriminant

$$
D^{2}\left[P_{n}(x)\right]=\left(2 n^{2} h_{n}\right)^{n} \frac{(-1)^{n} P_{n}^{2}\left(r_{+}\right) P_{n}^{2}\left(r_{-}\right)}{P_{n}^{2}(\alpha) P_{n}(1) P_{n}(-1) P_{n}(\beta) P_{n}(\gamma(n))} .
$$

The product $P_{n}(\beta) P_{n}\left(\gamma_{n}\right)$ is always positive since we know from 9 that $P_{n}(x)$ cannot have a zero in the interval $(\gamma(n), \beta)$. A similar calculation for the polynomials of the second kind gives

$$
D^{2}\left[Q_{n}(x)\right]=\left(2 n^{2} h_{n}\right)^{n-1} \frac{Q_{n}^{2}\left(r_{+}(n)\right) Q_{n}^{2}\left(r_{-}(n)\right)}{Q_{n}^{2}(1) Q_{n}^{2}(-1) Q_{n}^{2}(\beta) Q_{n}(\gamma(n)) Q_{n}(\alpha)} .
$$

Again, in this case we require that $Q_{n}(x)$ have no zeroes at the branch points.

These discriminants are valid for genus 1 . Indeed we know explicitly the values of $\gamma(n)$ and $r_{ \pm}(n)$ for $g=1$. It is clear from the derivations in this section that the equivalent expressions for general genus $g$ are

$$
D^{2}\left[P_{n}(x)\right]=\left(2 n^{2} h_{n}\right)^{n} \frac{(-1)^{n} \prod_{j=1}^{2 g} P_{n}^{2}\left(r_{j}(n)\right)}{P_{n}(1) P_{n}(-1) \prod_{j=1}^{g}\left[P_{n}\left(\beta_{j}\right) P_{n}\left(\gamma_{j}(n)\right) P_{n}^{2}\left(\alpha_{j}\right)\right]},
$$

and

$$
D^{2}\left[Q_{n}(x)\right]=\left(2 n^{2} h_{n}\right)^{n-1} \frac{\prod_{j=1}^{2 g} Q_{n}^{2}\left(r_{j}(n)\right)}{Q_{n}^{2}(1) Q_{n}^{2}(-1) \prod_{j=1}^{g}\left[Q_{n}^{2}\left(\beta_{j}\right) Q_{n}\left(\gamma_{j}(n)\right) Q_{n}\left(\alpha_{j}\right)\right]},
$$

where the $\left\{r_{j}(n)\right\}$ are the $2 g$ roots of the polynomial

$$
\left[\prod_{j=1}^{g}\left(x-\gamma_{j}(n)\right)\right]\left(n x^{g}-\sum_{j=0}^{g-1} c_{j}(n) x^{j}-\frac{1}{2} \sum_{j=1}^{g} \frac{y_{j}}{x-\gamma_{j}(n)}\right) .
$$

\section{Connection With polynomial mappings}

The theta function expressions for the recurrence coefficients $a_{n}$ and $b_{n}$ of the generalized Chebyshev polynomials are given in (1.8) and (1.9). Consider for the moment two different values of the recurrence coefficients, for example, $b_{n}$ and $b_{n+\mathfrak{K}}$, where $n$ and $\mathfrak{K}$ are both integers. If $\mathfrak{K}$ is chosen such that $\mathfrak{K} \hat{\mathbf{B}} \in \mathbb{Z}^{g}$, then from the periodicity properties of the theta functions we have

$$
b_{n+\mathfrak{K}}=b_{n} .
$$

From the expression for the $a_{n}$ coefficients, we will also have

$$
a_{n+\mathfrak{K}}=a_{n}, \quad n \geq 2 .
$$

When the entries of the vector $\hat{\mathbf{B}}$ are all rational numbers, we can always find an integer $\mathfrak{K}$ such that $\mathfrak{K} \hat{\mathbf{B}} \in \mathbb{Z}^{g}$. If we take the smallest integer that satisfies this requirement we will then have the smallest integer over which the recurrence coefficients themselves repeat, that is, the recurrence coefficients are periodic with period $\mathfrak{K}$. If at least one of the entries is an irrational number we are unable to find an integer $\mathfrak{K}$ that satisfies the periodicity requirement.

We will study the effect that this periodicity has on our previous constructions and use this to explain the connection between the generalized Chebyshev polynomials and non-trivial polynomial mappings [10]. Throughout this section $\mathfrak{K}$ is a 
given fixed integer. First consider $P_{n \mathfrak{K}}(x)$. From (1.5), and with the periodicity condition, we see that the $\theta$ factors cancel in equation (1.6), therefore

$$
P_{n \mathfrak{K}}(x)=\frac{\Delta_{n \mathfrak{K}}}{2}\left(e^{n \mathfrak{K}\left(\Omega\left(p_{x}\right)\right)}+e^{-n \mathfrak{K}\left(\Omega\left(p_{x}\right)\right)}\right),
$$

hence,

$$
P_{\mathfrak{K}}(x)=\frac{\Delta_{\mathfrak{K}}}{2}\left(e^{\mathfrak{K}\left(\Omega\left(p_{x}\right)\right)}+e^{-\mathfrak{K}\left(\Omega\left(p_{x}\right)\right)}\right) .
$$

Here,

$$
\Delta_{n \mathfrak{K}}=2 e^{-n \mathfrak{K} \chi_{0}} .
$$

Therefore,

$$
\begin{aligned}
P_{n \mathfrak{K}}(x) & =2^{n-1} \Delta_{n \mathfrak{K}} T_{n}\left(\frac{e^{\mathfrak{K} \Omega(x)}+e^{-\mathfrak{K} \Omega(x)}}{2}\right) \\
& =\Delta_{\mathfrak{K}}^{n} T_{n}\left(\frac{P_{\mathfrak{K}}(x)}{\Delta_{\mathfrak{K}}}\right),
\end{aligned}
$$

where $T_{n}(x)$ is the monic Chebyshev polynomial. From the expression for $h_{n}$ in (1.10) we see that

$$
h_{\mathfrak{K}}=2 e^{-2 \mathfrak{K} \chi_{0}} .
$$

It follows that

$$
\Delta_{\mathfrak{K}}=\sqrt{2 h_{\mathfrak{K}}}
$$

and therefore,

$$
P_{n \mathfrak{K}}(x)=\left(2 h_{\mathfrak{K}}\right)^{\frac{n}{2}} T_{n}\left(\frac{P_{\mathfrak{K}}(x)}{\sqrt{2 h_{\mathfrak{K}}}}\right) .
$$

The corresponding formula for the polynomials of the second kind is

$$
Q_{n \mathfrak{K}}(x)=\left(2 h_{\mathfrak{K}}\right)^{\frac{n-1}{2}} Q_{\mathfrak{K}}(x) U_{n}\left(\frac{P_{\mathfrak{K}}(x)}{\sqrt{2 h_{\mathfrak{K}}}}\right),
$$

where $U_{n}(x)$ is the Chebyshev polynomial of the second kind (degree $\left.n-1\right)$. Recall that

$$
\mathcal{E}_{n}\left(\mathfrak{p}_{x}\right) \tilde{\mathcal{E}}_{n}\left(\mathfrak{p}_{x}\right)=\frac{S_{g}(x ; n)}{\prod_{j=1}^{g}\left(x-\alpha_{j}\right)} .
$$

When $\mathfrak{K} \hat{\mathbf{B}} \in \mathbb{Z}^{g}$, we have

$$
\mathcal{E}_{n}\left(\mathfrak{p}_{x}\right) \tilde{\mathcal{E}}_{n}\left(\mathfrak{p}_{x}\right)=4 e^{-2 n \chi_{0}} .
$$

Therefore, since the $\gamma_{j}(n)$ are the zeroes of $S_{g}(x ; n)$ we must have $\gamma_{j}(\mathfrak{K})=\alpha_{j}$. It will be shown later in this section that the maximum value of $g$ permitted for the polynomials is $\mathfrak{K}-1$. Therefore, if we put $g=\mathfrak{K}-1$ and $n=\mathfrak{K}$ in (1.13) we see immediately that

$$
Q_{\mathfrak{K}}(x)=\prod_{j=1}^{\mathfrak{K}-1}\left(x-\alpha_{j}\right)
$$

Hence,

$$
Q_{n \mathfrak{K}}(x)=\left(2 h_{\mathfrak{K}}\right)^{\frac{n-1}{2}} U_{n}\left(\frac{P_{\mathfrak{K}}(x)}{\sqrt{2 h_{\mathfrak{K}}}}\right) \prod_{j=1}^{\mathfrak{K}-1}\left(x-\alpha_{j}\right) .
$$


In the language of (3.3), (4.5) becomes

$$
P_{n \mathfrak{K}}(x)=\frac{\left(\prod_{j=1}^{\mathfrak{K}} f_{+}(x, j)\right)^{n}+\left(\prod_{j=1}^{\mathfrak{K}} f_{-}(x, j)\right)^{n}}{2^{n}},
$$

and (4.6) becomes

$$
Q_{n \mathfrak{K}}(x)=\psi(x) \frac{\left(\prod_{j=1}^{\mathfrak{K}} f_{+}(x, j)\right)^{n}-\left(\prod_{j=1}^{\mathfrak{K}} f_{-}(x, j)\right)^{n}}{2^{n}} .
$$

Using the theta function expressions, we can verify the following:

$$
f_{+}(x, \mathfrak{K}+1)=\frac{\tilde{\mathcal{E}}_{\mathfrak{K}+1}\left(\mathfrak{p}_{x}\right)}{\tilde{\mathcal{E}}_{\mathfrak{K}}\left(\mathfrak{p}_{x}\right)}=\frac{\tilde{\Delta}_{\mathfrak{K}+1}}{\tilde{\Delta}_{\mathfrak{K}} \tilde{\Delta}_{1}} f_{+}(x, 1) .
$$

Similarly we can deduce that

and therefore,

$$
\frac{\tilde{\Delta}_{\mathfrak{K}+1}}{\tilde{\Delta}_{\mathfrak{K}} \tilde{\Delta}_{1}}=\frac{1}{2}
$$

$$
f_{ \pm}(x, \mathfrak{K}+1)=\frac{1}{2} f_{ \pm}(x, 1) .
$$

An analogous calculation shows that

$$
f_{ \pm}(x, \mathfrak{K}+j)=f_{ \pm}(x, j), \quad 1<j<\mathfrak{K},
$$

as we expect from looking at the explicit structure of the $f_{ \pm}(x, j)$.

If we know the period $\mathfrak{K}$, then we can use the $P_{\mathfrak{K}}(x)$ with (4.5) to generate every $\mathfrak{K}^{\text {th }}$ polynomial in the sequence. To obtain the intermediate polynomials $P_{n \mathfrak{K}+j}$ for $j=1, \ldots, \mathfrak{K}-1$ we use the fact that

$$
\mathcal{E}_{n \mathfrak{K}+j}\left(\mathfrak{p}_{x}\right)=\frac{1}{2}\left(\prod_{l=1}^{j} f_{+}(x ; l)\right) \mathcal{E}_{n \mathfrak{K}}\left(\mathfrak{p}_{x}\right)=\frac{1}{2} \mathcal{E}_{j}\left(\mathfrak{p}_{x}\right) \mathcal{E}_{n \mathfrak{K}}\left(\mathfrak{p}_{x}\right),
$$

which follows directly from the theta function representation of these functions, and therefore,

$$
P_{n \mathfrak{K}+j}(x)=\frac{1}{2}\left(P_{j}(x) P_{n \mathfrak{K}}(x)+\frac{1}{\psi^{2}(x)} Q_{j}(x) Q_{n \mathfrak{K}}(x)\right), \quad 1 \leq j<\mathfrak{K}, \quad n \geq 1 .
$$

Similarly,

$$
Q_{n \mathfrak{K}+j}(x)=\frac{1}{2}\left(P_{j}(x) Q_{n \mathfrak{K}}(x)+Q_{j}(x) P_{n \mathfrak{K}}(x)\right), \quad 1 \leq j<\mathfrak{K}, \quad n \geq 1 .
$$

So if we know the first $\mathfrak{K}$ polynomials of the first and second kind we can generate all of the remaining polynomials using (4.5), (4.6), (4.7) and (4.8).

From the theory of the classical Chebyshev polynomials we have

$$
T_{m}(x) T_{n}(x)=\left\{\begin{array}{l}
T_{m+n}(x)+\frac{1}{2^{2 n}} T_{m-n}(x), \quad m>n \neq 0, \\
T_{2 n}(x)+\frac{1}{2^{2 n-1}} T_{0}, \quad m=n .
\end{array}\right.
$$

Similarly,

$$
U_{m}(x) T_{n}(x)= \begin{cases}U_{m+n}(x)+\frac{1}{2^{2 n}} U_{m-n}(x), & m \geq n \neq 0 \\ U_{m+n}(x)-\frac{1}{2^{2 m}} U_{n-m}(x), & n \geq m \neq 0 .\end{cases}
$$

Analogous formulas to the ones above do not hold for all of the generalized Chebyshev polynomials. However, $P_{n \mathfrak{K}}$ play a similar role to the classical Chebyshev polynomials. 
Lemma 1. For any $m, n \in \mathbb{Z}_{+}$we have

$$
P_{n}(x) P_{m \mathfrak{K}}(x)= \begin{cases}P_{n+m \mathfrak{K}}(x)+\frac{h_{m \mathfrak{K}}}{2} P_{n-m \mathfrak{K}}(x), & n>m \mathfrak{K} \neq 0, \\ P_{n+m \mathfrak{K}}(x)+\frac{h_{n}}{2} P_{m \mathfrak{K}-n}(x), & m \mathfrak{K}>n \neq 0, \\ P_{2 m \mathfrak{K}}(x)+h_{m \mathfrak{K}} P_{0}(x), & n=m \mathfrak{K} .\end{cases}
$$

Proof. From section 1,

$$
P_{n}(x)=e^{-n \chi_{0}} \frac{\vartheta\left(\mathbf{u}^{+}\right)}{\vartheta\left(\mathbf{u}^{+}+n \hat{\mathbf{B}}\right)}\left[e^{n \Omega\left(\mathfrak{p}_{x}\right)} \frac{\vartheta\left(\mathbf{u}_{x}+n \hat{\mathbf{B}}\right)}{\vartheta\left(\mathbf{u}_{x}\right)}+e^{-n \Omega\left(\mathfrak{p}_{x}\right)} \frac{\vartheta\left(\mathbf{u}_{x}-n \hat{\mathbf{B}}\right)}{\vartheta\left(\mathbf{u}_{x}\right)}\right],
$$

and

Therefore,

$$
P_{m \mathfrak{K}}(x)=e^{-m \mathfrak{K} \chi_{0}}\left[e^{m \mathfrak{K} \Omega\left(\mathfrak{p}_{x}\right)}+e^{-m \mathfrak{K} \Omega\left(\mathfrak{p}_{x}\right)}\right] .
$$

$$
\begin{aligned}
P_{n}(x) P_{m \mathfrak{K}}(x)= & e^{-(n+m \mathfrak{K}) \chi_{0}} \frac{\vartheta\left(\mathbf{u}^{+}\right)}{\vartheta\left(\mathbf{u}^{+}+n \hat{\mathbf{B}}\right)}\left[e^{(n+m \mathfrak{K}) \Omega\left(\mathfrak{p}_{x}\right)} \frac{\vartheta\left(\mathbf{u}_{x}+n \hat{\mathbf{B}}\right)}{\vartheta\left(\mathbf{u}_{x}\right)}\right. \\
& +e^{-(n+m \mathfrak{K}) \Omega\left(\mathfrak{p}_{x}\right)} \frac{\vartheta\left(\mathbf{u}_{x}-n \hat{\mathbf{B}}\right)}{\vartheta\left(\mathbf{u}_{x}\right)} \\
& \left.+e^{(n-m \mathfrak{K}) \Omega\left(\mathfrak{p}_{x}\right)} \frac{\vartheta\left(\mathbf{u}_{x}+n \hat{\mathbf{B}}\right)}{\vartheta\left(\mathbf{u}_{x}\right)}+e^{-(n-m \mathfrak{K}) \Omega\left(\mathfrak{p}_{x}\right)} \frac{\vartheta\left(\mathbf{u}_{x}-n \hat{\mathbf{B}}\right)}{\vartheta\left(\mathbf{u}_{x}\right)}\right] .
\end{aligned}
$$

From the periodicity of the theta functions we may add the vector $\pm m \mathfrak{K} \hat{\mathbf{B}}$ to any of the arguments of the theta functions without changing the equality. We make this change in the argument of the theta functions in such a way that we are left with (4.9).

Similarly, for the polynomials of the second kind, we have

$$
Q_{n}(x) P_{m \mathfrak{K}}(x)=\left\{\begin{array}{l}
Q_{n+m \mathfrak{K}}(x)+\frac{h_{m \mathfrak{K}}}{2} Q_{n-m \mathfrak{K}}(x), \quad n \geq m \mathfrak{K} \neq 0 \\
Q_{n+m \mathfrak{K}}(x)-\frac{h_{n}}{2} Q_{m \mathfrak{K}-n}(x), \quad m \mathfrak{K} \geq n \neq 0
\end{array}\right\} .
$$

Combining (4.9) and (4.7) we obtain

$$
P_{n \mathfrak{K}-j}(x)=\frac{1}{h_{j}}\left(P_{n k}(x) P_{j}(x)-\frac{Q_{n k}(x) Q_{j}(x)}{\psi^{2}(x)}\right), \quad j=1, \ldots, \mathfrak{K}-1 .
$$

Similarly,

$$
Q_{n \mathfrak{K}-j}(x)=\frac{1}{h_{j}}\left(Q_{n \mathfrak{K}}(x) P_{j}(x)-P_{n \mathfrak{K}}(x) Q_{j}(x)\right) .
$$

From (1.8) it follows from the periodicity properties of the theta functions that

$$
a_{j}=a_{\mathfrak{K}-j+1}, \quad j=1, \ldots, \mathfrak{K}-1 .
$$

The $b_{n}$ also have a similar behaviour, and one can show that

$$
b_{j}=b_{\mathfrak{K}-j+2}, \quad j=1, \ldots, \mathfrak{K}-1 .
$$

However, this is not obvious from the form of the recurrence coefficients given in (1.9). To prove the above equality we start with the three term recurrence relation (1.4) with $n=\mathfrak{K}-j+1$ and $x=1$, so that

$$
P_{\mathfrak{K}-j+1}(1)=P_{\mathfrak{K}-j+2}(1)+b_{\mathfrak{K}-j+2} P_{\mathfrak{K}-j+1}(1)+a_{\mathfrak{K}-j+1} P_{\mathfrak{K}-j}(1) .
$$

From the expression for $P_{n \mathfrak{K}-j}(x)$ we have

$$
P_{n \mathfrak{K}-j}(1)=\frac{P_{n \mathfrak{K}}(1) P_{j}(1)}{h_{j}} .
$$


Substituting this into the three term recurrence relation we obtain

$$
P_{j-1}(1)=P_{j}(1)+b_{\mathfrak{K}-j+2} P_{j-1}(1)+a_{j-1} P_{j-2}(1) .
$$

Comparing this with (1.4) at $x=1$ and $n=j-1$ we see that $b_{\mathfrak{K}-j+2}=b_{j}$.

Theorem 4.1. If the set $E$ is chosen such that the vector $\mathfrak{K} \hat{\mathbf{B}} \in \mathbb{Z}^{g}$, then $E$ is the inverse image of $[-1,1]$ under the mapping $\frac{P_{\mathfrak{\Omega}}(x)}{\Delta_{\mathfrak{K}}}$.

Proof. Since $\mathfrak{K} \hat{\mathbf{B}} \in \mathbb{Z}^{g}$, we have

$$
i \mathfrak{K} \Omega\left(\beta_{j}\right)=i \mathfrak{K} \Omega\left(\alpha_{j}\right)=m \pi \quad \text { for some } m \in \mathbb{Z},
$$

and, from (10.13) in 9 ,

$$
i \mathfrak{K} \Omega(-1)=-\mathfrak{K} \pi
$$

which also can be shown by a contour integral on the contour $\Lambda$ in section 2 . From (4.2), we have

$$
\frac{P_{\mathfrak{K}}(x)}{\Delta_{\mathfrak{K}}}=\cos i \mathfrak{K} \Omega(x) .
$$

For $x \in E, \Omega(x) \in \mathbb{R}$ so $\left|\frac{P_{\mathfrak{K}}(x)}{\Delta_{\mathfrak{K}}}\right|$ attains the value $1, \mathfrak{K}-1-g$ times on the interior of $E$. Since there are $g$ stationary points of the polynomial corresponding to one in each gap, the polynomial has the mapping properties stated in the theorem.

In figure 1 we show an example of a mapping polynomial for $\mathfrak{K}=3$. We now consider some examples of sequences of orthogonal polynomials generated via the polynomial mappings $P_{\mathfrak{K}}(x)$. We choose small values of $\mathfrak{K}$ and investigate. $\mathfrak{K}=1$ corresponds to the Chebyshev polynomials as the recurrence coefficients are constant. For $\mathfrak{K}=2$, the mapping polynomial,

$$
M_{2}(x)=\frac{1}{\Delta_{2}} P_{2}(x)=\frac{1}{\Delta_{2}}\left(x^{2}+a x+b_{2}\right),
$$

satisfies the following conditions:

$$
\begin{aligned}
& \frac{1}{\Delta_{2}} P_{2}(-1)=\frac{1}{\Delta_{2}} P_{2}(1)=1, \\
& \frac{1}{\Delta_{2}} P_{2}(\alpha)=\frac{1}{\Delta_{2}} P_{2}(\beta)=-1 .
\end{aligned}
$$

This implies that $M_{2}(x)$ has the following form:

$$
M_{2}(x)=\frac{1}{\Delta_{2}} x^{2}+\left(1-\frac{1}{\Delta_{2}}\right),
$$

with

$$
\begin{aligned}
\alpha^{2} & =\beta^{2}, \\
\Delta_{2} & =\frac{1-\alpha^{2}}{2}=\frac{1-\beta^{2}}{2} .
\end{aligned}
$$

Therefore,

$$
M_{2}(x)=\frac{2}{1-\alpha^{2}} x^{2}+\left(1-\frac{2}{1-\alpha^{2}}\right),
$$

with the condition on the branch points, $\alpha=-\beta$. The condition above tells us which sets $E$ arise from a polynomial mapping of degree 2 . These are the same sets that give rise to periodic recurrence coefficients with period 2 . To obtain the recurrence coefficients for these polynomials, we can substitute $\beta=-\alpha$ into the 


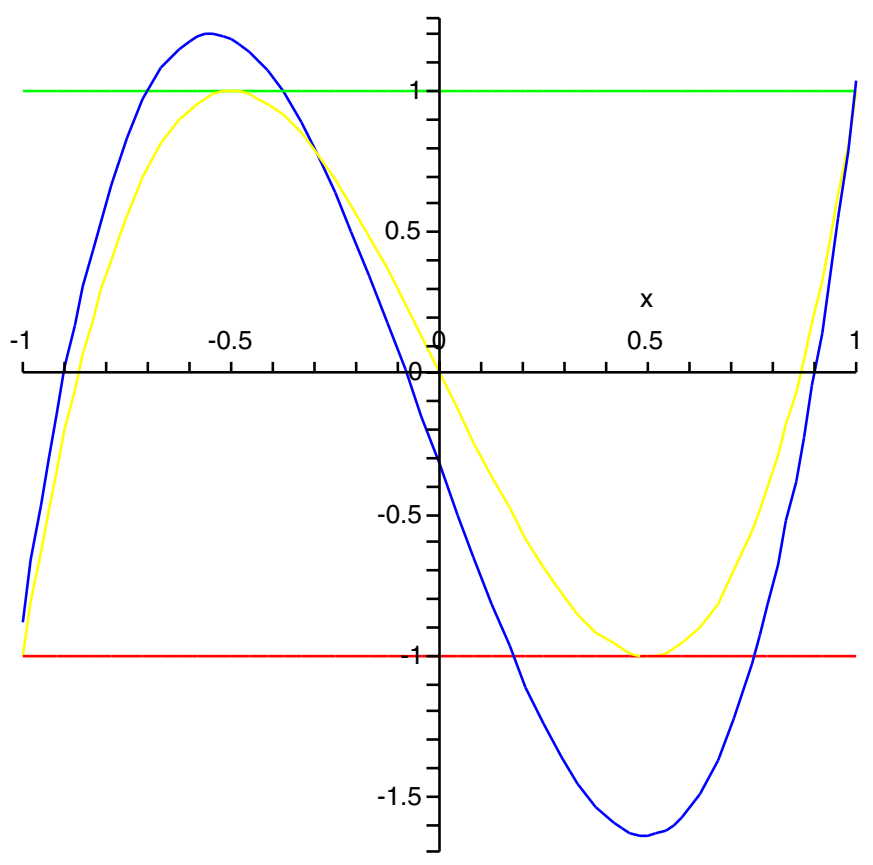

Figure 1. General form of $M_{3}(x)$ compared with the Chebyshev polynomial of degree 3 . The branch points are where the polynomial crosses the lines $y=-1$ and $y=1$.

general genus 1 recurrence coefficients. The general expressions for the recurrence coefficients can be obtained in one of two ways. Appendix A shows how we can manipulate the theta function expressions for the recurrence coefficients into expressions involving the Jacobian elliptic functions. We are then able to invert the functions and in principle obtain the recurrence coefficients for any $n$ in terms of the branch points only. In this case we only need to find the first two since the others repeat. The second way to find the recurrence coefficients is to use the non-linear difference equations derived in section 2. Once we have the initial conditions we can iterate the non-linear difference equations to find the remaining coefficients. This method is more practical when we are considering higher periodicity. In any case, the recurrence coefficients are found to be

$$
\begin{aligned}
b_{n} & =(-1)^{n} \alpha, \\
a_{n} & =\frac{1}{4}\left(1-\alpha^{2}\right), \quad n \geq 2 .
\end{aligned}
$$

With these coefficients, our polynomials become

$$
P_{2 n}(x)=\left(\Delta_{2}\right)^{n} T_{n}\left(\frac{P_{2}(x)}{\Delta_{2}}\right)
$$


and

$$
P_{2 n+1}(x)=\frac{(x+\alpha)}{2}\left(\Delta_{2}\right)^{n}\left(T_{n}\left(\frac{P_{2}(x)}{\Delta_{2}}\right)+\frac{\left(x^{2}-1\right)}{\Delta_{2}} U_{n}\left(\frac{P_{2}(x)}{\Delta_{2}}\right)\right) .
$$

Also the auxiliary polynomials become

$$
\begin{aligned}
& S_{1}(x, n)=2 h_{n}\left(x+(-1)^{n+1} \alpha\right), \\
& G_{2}(x, n)=h_{n-1}(x+\alpha)(x-\alpha) .
\end{aligned}
$$

From (3.10) we have

$$
f_{1}(x ; n)=\frac{1}{2}\left(\frac{1}{x+(-1)^{n+1} \alpha}-\frac{1}{x-\alpha}\right) .
$$

To evaluate $f_{2}(x ; n)$ we must know the $c_{0}(n)$ and $\gamma(n)$. For genus 1 polynomials we have from [9],

$$
c_{0}(n)=p_{1}(n)+n \frac{\alpha+\beta}{2}+\frac{\gamma(n)-\alpha}{2},
$$

where $p_{1}(n)$ is the coefficient of $x^{n-1}$ in $P_{n}(x)$. In this case,

$$
\begin{aligned}
& p_{1}(2 n)=0, \\
& p_{1}(2 n-1)=\alpha .
\end{aligned}
$$

Therefore,

$$
c_{0}(n)=0 .
$$

Since $\gamma(n)$ is the zero of $S_{g}(x, n)$,

$$
\gamma(n)=(-1)^{n} \alpha
$$

and

$$
f_{2}(x ; n)=\frac{n x}{x-\alpha} .
$$

For even $n$, the discriminant becomes $(n=2 m)$

$$
D\left[P_{2 m}(x)\right]=(-1)^{m} 2^{-(2 m-1)(2 m-2)-m}(2 m)^{2 m}\left(1-\alpha^{2}\right)^{m(2 m-1)} T_{m}\left(\frac{\alpha^{2}+1}{\alpha^{2}-1}\right)
$$

and for odd $n(n=2 m+1)$

$$
\begin{aligned}
D\left[P_{2 m+1}(x)\right]=(-1)^{m} 2^{-4 m^{2}+m-1}(2 m+1)^{2 m}\left(1-\alpha^{2}\right)^{m(2 m+1)} & \\
& {\left[T_{m}\left(\frac{\alpha^{2}+1}{\alpha^{2}-1}\right)-\frac{2}{1-\alpha^{2}} U_{m}\left(\frac{\alpha^{2}+1}{\alpha^{2}-1}\right)\right] . }
\end{aligned}
$$

The discriminant for the case when $n$ is odd cannot be obtained directly from (3.15) since the $P_{2 n+1}(x)$ have zeroes at $x=-\alpha$. The problem is that of evaluating $P_{2 n+1}^{\prime}(-\alpha)$. In general evaluating the derivative at one of the branch points is not straightforward. However, in this case it can be verified that

$$
P_{2 n+1}^{\prime}(-\alpha)=2 f_{2}(-\alpha ; 2 n+1) Q_{2 n+1}(-\alpha),
$$

whereas, for the zeroes that do not lie on the branch points we have

$$
P_{2 n+1}^{\prime}\left(x_{j, 2 n+1}\right)=f_{2}\left(x_{j, 2 n+1} ; 2 n+1\right) Q_{2 n+1}\left(x_{j, 2 n+1}\right)
$$

and the factor of 2 does not appear. It is for this reason that the expression given in (3.15) remains valid in this case if we insert a factor of 2 . 
The polynomials in this example can also be shown to satisfy the following differential equation:

$$
P_{n}^{\prime \prime}-\frac{x^{4}-\alpha^{2}}{x\left(x^{2}-\alpha^{2}\right)\left(1-x^{2}\right)} P_{n}^{\prime}+\frac{n^{2} x^{2}}{\left(1-x^{2}\right)\left(x^{2}-\alpha^{2}\right)} P_{n}=0 \quad \text { for even } n,
$$

and

$$
P_{n}^{\prime \prime}-\frac{x^{4}-2 \alpha x\left(1-x^{2}\right)+\alpha^{2}}{x\left(x^{2}-\alpha^{2}\right)\left(1-x^{2}\right)} P_{n}^{\prime}+\frac{n^{2} x^{4}-2 x \alpha+\left(n^{2}+1\right) x^{3} \alpha-\alpha^{2}}{x\left(1-x^{2}\right)(x-\alpha)(x+\alpha)^{2}} P_{n}=0 \text { odd } n .
$$

For the next example we choose $\mathfrak{K}=3$. Therefore our polynomials can be described in terms of a polynomial mapping of degree 3 . The highest genus permitted in this case is 2 . This corresponds to three disjoint intervals. Genus one cases arise if we close one of the gaps. In the last example we saw that the only sets that gave rise to a period of 2 were symmetric around the origin, that is, $\alpha=-\beta$. The aim of this example is to determine which sets give rise to recurrence coefficients with period 3 . We determine the recurrence coefficients, the $\gamma$ points and under a certain condition, the $c_{j}(n)$. We have a mapping polynomial $M_{3}(x)$ with leading coefficient $\frac{1}{\Delta_{3}}$ that satisfies the following equations:

$$
\begin{aligned}
& M_{3}( \pm 1)= \pm 1, \\
& M_{3}\left(\alpha_{1}\right)=M_{3}\left(\beta_{1}\right)=1, \\
& M_{3}\left(\alpha_{2}\right)=M_{3}\left(\beta_{2}\right)=-1 .
\end{aligned}
$$

Solving these six equations we have

$$
M_{3}(x)=\frac{x^{3}}{\Delta_{3}}+a x^{2}+\left(1-\frac{1}{\Delta_{3}}\right) x-a,
$$

with

$$
a=\frac{-\left(\beta_{1}+\alpha_{1}+1\right)}{\left(\beta_{1}+1\right)\left(\alpha_{1}+1\right)}=\frac{-\left(\alpha_{2}+\beta_{2}-1\right)}{\left(\beta_{2}-1\right)\left(\alpha_{2}-1\right)}
$$

and

$$
\Delta_{3}=\left(\beta_{1}+1\right)\left(\alpha_{1}+1\right)=\left(\beta_{2}-1\right)\left(\alpha_{2}-1\right) .
$$

Since we can characterize the mapping polynomial in terms of two branch points, we have a system with two unknowns. We describe the system in terms of $\alpha_{1}$ and $\beta_{1}$. The form of the mapping polynomial restricts $\alpha_{1}$ and $\beta_{1}$ to certain intervals on $[-1,1]$. If we eliminate $\alpha_{2}$ from (4.11) and (4.12) we get

$$
\beta_{2}=\frac{\alpha_{1}+\beta_{1}+2 \pm \sqrt{\left(\beta_{1}-\alpha_{1}\right)^{2}-4\left(\alpha_{1}+\beta_{1}+1\right)}}{2}
$$

hence,

$$
\left(\alpha_{1}-\beta_{1}\right)^{2}-4\left(\alpha_{1}+\beta_{1}+1\right) \geq 0 .
$$

Therefore we have

$$
\alpha_{1} \leq \beta_{1}+2-4 \sqrt{\frac{\beta_{1}+1}{2}} .
$$

This inequality, coupled with the inequality

$$
\alpha_{1} \leq \beta_{1},
$$


implies that $\alpha_{1}$ lies on the interval $\left[-1,-\frac{1}{2}\right]$. Therefore $\beta_{1}$ lies on the interval $\left[\alpha_{1}, \beta_{1, \max }\right]$ where

$$
\beta_{1, \max }=2+\alpha_{1}-4 \sqrt{\frac{\alpha_{1}+1}{2}} .
$$

The value of $\beta_{1, \max }$ follows from (4.13) with the equality sign. With these conditions on $\alpha_{1}$ and $\beta_{1}$ we have

$$
\alpha_{2}=1+\frac{\alpha_{1}+\beta_{1}}{2}-\frac{1}{2} \sqrt{\left(\beta_{1}-\alpha_{1}\right)^{2}-4\left(\alpha_{1}+\beta_{1}+1\right)}
$$

and

$$
\beta_{2}=1+\frac{\alpha_{1}+\beta_{1}}{2}+\frac{1}{2} \sqrt{\left(\beta_{1}-\alpha_{1}\right)^{2}-4\left(\alpha_{1}+\beta_{1}+1\right)} .
$$

For $g=2$ we evaluate the first three recurrence coefficients in the most general case in terms of $\alpha_{1}, \alpha_{2}, \beta_{1}$ and $\beta_{2}$, using (1.13) and (1.14). Then we substitute for $\alpha_{2}$ and $\beta_{2}$ using (4.14) and (4.15) and find

$$
\begin{aligned}
& a_{1}=\frac{1}{2}\left(1+\alpha_{1}\right)\left(2-\alpha_{1}+\beta_{1}+\sqrt{\left(\beta_{1}-\alpha_{1}\right)^{2}-4\left(1+\alpha_{1}+\beta_{1}\right)}\right) \\
& a_{2}=\frac{1}{16}\left(\alpha_{1}-\beta_{1}-2+\sqrt{\left(\beta_{1}-\alpha_{1}\right)^{2}-4\left(1+\alpha_{1}+\beta_{1}\right)}\right)^{2} \\
& a_{3}=\frac{1}{4}\left(1+\alpha_{1}\right)\left(2-\alpha_{1}+\beta_{1}+\sqrt{\left(\beta_{1}-\alpha_{1}\right)^{2}-4\left(1+\alpha_{1}+\beta_{1}\right)}\right),
\end{aligned}
$$

and

$$
\begin{aligned}
& b_{1}=\frac{1}{2}\left(\beta_{1}-\alpha_{1}+\sqrt{\left(\beta_{1}-\alpha_{1}\right)^{2}-4\left(1+\alpha_{1}+\beta_{1}\right)}\right) \\
& b_{2}=\frac{1}{4}\left(2+3 \alpha_{1}+\beta_{1}-\sqrt{\left(\beta_{1}-\alpha_{1}\right)^{2}-4\left(1+\alpha_{1}+\beta_{1}\right)}\right) \\
& b_{3}=\frac{1}{4}\left(2+3 \alpha_{1}+\beta_{1}-\sqrt{\left(\beta_{1}-\alpha_{1}\right)^{2}-4\left(1+\alpha_{1}+\beta_{1}\right)}\right) .
\end{aligned}
$$

We also find that, for $n \geq 2$,

$$
\begin{aligned}
\gamma_{1,2}(n) & =\frac{1}{2}\left(1+\alpha_{1}+\beta_{1}-b_{n+1}\right. \\
& \left.\mp \sqrt{1-2\left(\alpha_{1}+\beta_{1}\right)+\left(\alpha_{1}-\beta_{1}\right)^{2}-4\left(a_{n}+a_{n+1}\right)+2 b_{n+1}\left(1+\alpha_{1}+\beta_{1}-\frac{3}{2} b_{n+1}\right)}\right) .
\end{aligned}
$$

Expressions for the polynomials are easily obtained from our equations in the previous sections. Expressions for the discriminant and the differential equation can in principle be obtained using the constants that we have derived in this section.

A highly symmetric case corresponding to $g=2$ arises when $\beta_{1}+\alpha_{1}=-1$. With this, the $a$ defined by (4.11) is 0 , which implies that

$$
\alpha_{2}+\beta_{2}=1 \text {. }
$$

Substituting these into (4.12), gives

$$
\beta_{2}=1+\beta_{1} .
$$

This case corresponds to the first gap being symmetric about the point $-\frac{1}{2}$ and the second gap being symmetric about the point $\frac{1}{2}$. In this case the square roots 
appearing in the recurrence coefficients vanish leaving (with $\alpha=\alpha_{1}$ )

$$
\begin{aligned}
& a_{1}=-2 \alpha(1+\alpha), \\
& a_{2}=\frac{1}{4}, \\
& a_{3}=-\alpha(1+\alpha)
\end{aligned}
$$

and

$$
\begin{aligned}
& b_{1}=-(1+2 \alpha), \\
& b_{2}=\frac{1}{2}+\alpha, \\
& b_{3}=\frac{1}{2}+\alpha .
\end{aligned}
$$

Also,

$$
G_{3}(x, n)=h_{n-1}\left(x^{3}+\left(2 a_{n}-1-\alpha-\alpha^{2}\right) x+2 a_{n}\left(b_{n}+b_{n+1}\right)\right)
$$

and

$$
S_{2}(x, n)=2 h_{n}\left(x^{2}+b_{n+1} x+a_{n}+a_{n+1}+b_{n+1}^{2}-1-\alpha-\alpha^{2}\right) .
$$

This gives

$$
\gamma_{1,2}(n)=\frac{-b_{n+1} \mp \sqrt{b_{n+1}^{2}-4\left(a_{n}+a_{n+1}+b_{n+1}^{2}-1-\alpha-\alpha^{2}\right)}}{2} .
$$

The first three polynomials are

$$
\begin{aligned}
& P_{1}(x)=x+2 \alpha+1, \\
& P_{2}(x)=x^{2}+\left(\alpha+\frac{1}{2}\right) x-\frac{1}{2}, \\
& P_{3}(x)=x^{3}-\left(1+\alpha+\alpha^{2}\right) x .
\end{aligned}
$$

From (3.9), (3.10) and (3.12) we can calculate $f_{1}(x ; n)$ and $f_{2}(x ; n)$.

Returning to the previous non-symmetric genus 2 example, we add the restriction that one of the gaps is closed. We take the case where $\alpha_{2}=\beta_{2}$. In this situation (4.11) and (4.12) simplify to

$$
a=\frac{-\left(\beta_{1}+\alpha_{1}+1\right)}{\left(\beta_{1}+1\right)\left(\alpha_{1}+1\right)}=\frac{-\left(2 \alpha_{2}-1\right)}{\left(\alpha_{2}-1\right)^{2}},
$$

and

$$
\Delta_{3}=\left(\beta_{1}+1\right)\left(\alpha_{1}+1\right)=\left(\alpha_{2}-1\right)^{2} .
$$

Therefore, eliminating $\alpha_{2}$ from the above we obtain

$$
\left(\beta_{1}+1\right)\left(\alpha_{1}+1\right)=\left(\frac{\beta_{1}+\alpha_{1}}{2}\right)^{2}
$$

with the solutions

$$
\beta_{1}=\alpha_{1}+2 \pm 4 \sqrt{\frac{\alpha_{1}+1}{2}}
$$

Since $\alpha_{1} \leq \beta_{1}$ we must have

$$
\beta_{1}=\alpha_{1}+2-4 \sqrt{\frac{\alpha_{1}+1}{2}} .
$$

This relation governs the variation of $\beta_{1}$ as $\alpha_{1}$ varies over the interval $\left[-1,-\frac{1}{2}\right]$. It describes a contour in the $\left(\alpha_{1}, \beta_{1}\right)$ plane. Figure 2 shows a graphical representation 
of this contour with similar curves for higher values of $\mathfrak{K}$. Had we chosen the condition $\alpha_{1}=\beta_{1}$ for the other $g=1$ case we would have obtained

$$
\beta_{2}=-2+\alpha_{2}+4 \sqrt{\frac{1-\alpha_{2}}{2}},
$$

where $\alpha_{2}$ varies over $\left[-1, \frac{1}{2}\right]$. Using the method outlined in the Appendix we are able to compute the recurrence coefficients for $g=1$ explicitly. From now on we refer to $\alpha_{1}$ and $\beta_{1}$ as just $\alpha$ and $\beta$ respectively. Upon substituting for $\beta$ given by (4.18) into the general recurrence coefficients we obtain the following:

$$
\begin{aligned}
& a_{1}=2(\alpha+1)\left(1-\sqrt{\frac{1+\alpha}{2}}\right), \\
& a_{2}=\frac{\alpha+3}{2}-2 \sqrt{\frac{\alpha+1}{2}} \\
& a_{3}=(\alpha+1)\left(1-\sqrt{\frac{1+\alpha}{2}}\right)
\end{aligned}
$$

and

$$
\begin{aligned}
& b_{1}=1-2 \sqrt{\frac{1+\alpha}{2}} \\
& b_{2}=(\alpha+1)-\sqrt{\frac{1+\alpha}{2}}, \\
& b_{3}=(\alpha+1)-\sqrt{\frac{1+\alpha}{2}} .
\end{aligned}
$$

The recurrence coefficients repeat in blocks of three, however we must remember that $a_{3 n+1}=\frac{a_{1}}{2}, \quad n \geq 1$. Furthermore,

$$
\begin{aligned}
c_{0}(3 n) & =n\left(\alpha-2 \sqrt{\frac{1+\alpha}{2}}\right), \\
c_{0}(3 n+1) & =\frac{1}{2}\left((1+2 n) \alpha-(1+4 n) \sqrt{\frac{1+\alpha}{2}}\right), \\
c_{0}(3 n+2) & =\frac{1}{2}\left((1+2 n) \alpha-(3+4 n) \sqrt{\frac{1+\alpha}{2}}\right),
\end{aligned}
$$

and

$$
\begin{aligned}
\gamma(3 n) & =\alpha, \\
\gamma(3 n+1) & =-\sqrt{\frac{1+\alpha}{2}}, \\
\gamma(3 n+2) & =-\sqrt{\frac{1+\alpha}{2}} .
\end{aligned}
$$

These constants, listed above, allow one to calculate the differential equations and the discriminant given in the previous section. 
General $\mathfrak{K}$. For notational convenience we now label as $E_{\mathfrak{K}}$ the sets $E$ that give rise to period $\mathfrak{K}$ recurrence coefficients. Equivalently these are the sets for which the minimum degree of polynomial mapping from $E_{\mathfrak{K}}$ onto $[-1,1]$ is $\mathfrak{K}$. From our earlier examples,

$$
\begin{aligned}
& E_{1}=[-1,1], \\
& E_{2}=[-1, \alpha] \cup[-\alpha, 1], \quad \alpha \in[-1,0], \\
& E_{3}=[-1, \alpha] \cup[\beta, u(\alpha, \beta)] \cup[v(\alpha, \beta), 1], \quad \alpha \in\left[-1,-\frac{1}{2}\right], \quad \beta \in\left[\alpha, \beta_{\max }\right],
\end{aligned}
$$

where

$$
\begin{aligned}
& u(\alpha, \beta)=1+\frac{\alpha+\beta}{2}-\frac{1}{2} \sqrt{(\beta-\alpha)^{2}-4(\alpha+\beta+1)}, \\
& v(\alpha, \beta)=1+\frac{\alpha+\beta}{2}+\frac{1}{2} \sqrt{(\beta-\alpha)^{2}-4(\alpha+\beta+1)} .
\end{aligned}
$$

Each set $E_{\mathfrak{K}}$ is parametrized in terms of $\mathfrak{K}-1$ variables. As $\mathfrak{K}$ increases it becomes intractable to solve explicitly the relationships between the branch points. However, the branch points are constrained on certain curves in $[-1,1]^{2 g}$ where the co-ordinate axes of the $2 g$ cube label the position of the branch points.

In what follows, we describe the constraints on the mapping polynomial. This will provide a set of equations that define the curves of constant periodicity in the $2 g$ cube. We also show how to determine the smallest $\mathfrak{K}$ for a given configuration of the branch points.

For general $\mathfrak{K}$, the $2 \mathfrak{K}$ conditions imposed on the mapping polynomial,

$$
M_{\mathfrak{K}}(x)=a_{\mathfrak{K}} x^{\mathfrak{K}}+a_{\mathfrak{K}-1} x^{\mathfrak{K}-1}+\ldots+a_{1} x+a_{0},
$$

are

$$
\begin{aligned}
& M_{\mathfrak{K}}(-1)=(-1)^{\mathfrak{K}}, \\
& M_{\mathfrak{K}}(1)=1, \\
& M_{\mathfrak{K}}\left(\alpha_{j}\right)=M_{\mathfrak{K}}\left(\beta_{j}\right)=(-1)^{\mathfrak{K}+j}, \quad j=1, \ldots, \mathfrak{K}-1 .
\end{aligned}
$$

Therefore $\mathfrak{K}+1$ of these will determine the coefficients in terms of $\mathfrak{K}+1$ branch points. Using the first two equations of (4.20) and any $\mathfrak{K}-1$ of the remaining ones we can solve for the coefficients of the mapping polynomial in terms of $1,-1$ and $\mathfrak{K}-1$ free parameters. The other $\mathfrak{K}-1$ branch points are related to the free parameters by the remaining equations of (4.20). These equations determine a surface in the $2 g$ cube. The surface obtained in this way will correspond to maximum $g=\mathfrak{K}-1$. Closing one of the gaps, that is, setting $\alpha_{j}=\beta_{j}$ for some $j \leq \mathfrak{K}-1$, will determine surfaces corresponding to that value of $\mathfrak{K}$ for smaller values of $g$.

If we close all of the gaps by setting $\alpha_{j}=\beta_{j}$ for all $j=1, \ldots, \mathfrak{K}-1$, then the mapping polynomial becomes

$$
M_{\mathfrak{K}}(x)=2^{\mathfrak{K}-1} T_{\mathfrak{K}}(x) .
$$

If we denote by $\tilde{x}_{j}$ the point where $\alpha_{j}=\beta_{j}$, then we must have

$$
2^{\mathfrak{K}-1} T_{\mathfrak{K}}\left(\tilde{x}_{j}\right)= \pm 1
$$

from the properties of the mapping polynomial. Since $T_{n}(x)=2^{1-n} \cos (n \theta), \quad x=$ $\cos (\theta)$ we see that the set of points $\left\{\tilde{x}_{j}\right\}_{j=1}^{\mathfrak{K}-1}$ are the $\mathfrak{K}-1$ stationary points of the Chebyshev polynomial of degree $\mathfrak{K}$. Therefore, each gap i.e. $\left(\alpha_{i}, \beta_{i}\right)_{i=1}^{\mathfrak{K}-1}$ can be said 
to be 'centred' (not necessarily symmetrically) around one of the stationary points of the Chebyshev polynomial of degree $\mathfrak{K}$. The most simple example of this is the $\mathfrak{K}=2$ case where the gap is centred around the point $x=0$. In the $\mathfrak{K}=3, g=2$ case, the two gaps are centred around $x=\frac{1}{2}$ and $x=-\frac{1}{2}$. The stationary points of $T_{4}(x)$ are $x=-\frac{1}{\sqrt{2}}, x=0$, and $x=\frac{1}{\sqrt{2}}$. So for a polynomial mapping of degree 4 we have three distinct $g=1$ cases corresponding to one gap which is opened around one of the stationary points.

Consider now the $\mathfrak{K}=2$ mapping polynomial, $\frac{P_{2}(x)}{\Delta_{2}}$, which maps $[-1, \alpha] \cup[-\alpha, 1]$ to $[-1,1]$. From (4.5),

$$
P_{4}(x)=\Delta_{2}^{2} T_{2}\left(\frac{P_{2}(x)}{\Delta_{2}}\right)
$$

Since, $\Delta_{2}^{2}=2 \Delta_{4}$, and from the fact that the classical Chebyshev polynomials map $[-1,1]$ to $[-1,1]$, we see that $\frac{P_{4}(x)}{\Delta_{4}}$ also maps $[-1, \alpha] \cup[-\alpha, 1]$ to $[-1,1]$. Therefore, it is this configuration of the branch points which corresponds to the $\mathfrak{K}=4, g=1$ case where the gap is centered around $x=0$. Indeed for any mapping polynomial of even degree, one of the $g=1$ cases corresponds to this configuration, since a Chebyshev polynomial of even degree has a stationary point at zero.

The same reasoning as above applies for general $\mathfrak{K}$. That is, if $\frac{P_{\mathfrak{K}}(x)}{\Delta_{\mathfrak{K}}}$ is a mapping polynomial for a certain configuration of the branch points, then so are the polynomials $\frac{P_{n \Re}(x)}{\Delta_{n \Re}}$. Therefore amongst all of the configurations that correspond to a mapping of degree $n \mathfrak{K}$, there can be found those that correspond to degree $j \mathfrak{K}$, where $j$ divides $n$. If $\tilde{x}_{l}$ is a stationary point of $\widehat{T}_{\mathfrak{K}}(x):=2^{\mathfrak{K}-1} T_{\mathfrak{K}}(x)$, then

$$
\left|\widehat{T}_{\mathfrak{K}}\left(\tilde{x}_{l}\right)\right|=1
$$

and

$$
\left|\widehat{T}_{j \mathfrak{K}}\left(\tilde{x}_{l}\right)\right|=1,
$$

which indicates that $\tilde{x}_{l}$ is also a stationary point of $\widehat{T}_{j \mathfrak{K}}(x)$. For example $\widehat{T}_{12}(x)$ has amongst its stationary points, the stationary points of $\widehat{T}_{6}(x), \widehat{T}_{4}(x), \widehat{T}_{3}(x)$ and $\widehat{T}_{2}(x)$.

So as an example, a mapping polynomial of degree 12 will give rise to eleven $g=1$, single parameter cases of the generalized Chebyshev polynomials. Six of these will already be the same as those that arise from a mapping of degree 6 and four will be the same as those that arise from a mapping of degree 4 . All of these cases cover those that arise from mappings of degree 2 and 3 . Therefore a mapping of degree 12 will give rise to two $g=1$ cases that cannot be generated from polynomial mappings of lower degree.

The upshot of the above discussion is that we can uniquely label each surface in the $2 g$ cube by a set of points corresponding to the stationary points of the Chebyshev polynomials of any degree. We do this in the following way.

If we denote by $s_{l}$ the ordered set of all stationary points of the Chebyshev polynomial of degree 1 , then we can label a surface by taking any subset of the $s_{l}$. The number of elements in a subset is the number of gaps $g$ in the interval $[-1,1]$. 
For example,

$$
\begin{aligned}
& s_{2}=\{0\}, \\
& s_{3}=\left\{-\frac{1}{2}, \frac{1}{2}\right\}, \\
& s_{4}=\left\{-\frac{1}{\sqrt{2}}, 0, \frac{1}{\sqrt{2}}\right\}, \\
& s_{5}=\left\{-\frac{1}{4}(\sqrt{5}+1),-\frac{1}{4}(\sqrt{5}-1), \frac{1}{4}(\sqrt{5}-1), \frac{1}{4}(\sqrt{5}+1)\right\} .
\end{aligned}
$$

Therefore, the set

$$
\left\{-\frac{1}{\sqrt{2}}, \frac{1}{\sqrt{2}}\right\}
$$

corresponds to the $\mathfrak{K}=4 g=2$ surface where both gaps are centred around the two points $-\frac{1}{\sqrt{2}}$ and $\frac{1}{\sqrt{2}}$. This is a two parameter curve. Those curves shown in figure 2 can be labelled by one number which is a stationary point of a Chebyshev polynomial. It is clear from the discussion so far that the number $\mathcal{N}$ given by

$$
\mathcal{N}=\left(\begin{array}{c}
\mathfrak{K}-1 \\
g
\end{array}\right),
$$

is the number of surfaces in the $2 g$ cube associated with the integer $\mathfrak{K}$. So far, we have discussed in general terms how to describe the surfaces corresponding to a particular value of $\mathfrak{K}$. On the other hand, the problem of finding $\mathfrak{K}$ from a given set $E$ appears to be a very difficult numerical one. The condition for periodicity of the recurrence coefficients is

$$
\mathfrak{K} \hat{\mathbf{B}} \in \mathbb{Z}^{g} \backslash\left\{0^{g}\right\},
$$

where

$$
\hat{\mathbf{B}}_{j}=\frac{1}{2 \pi i} \int_{b_{j}} d \Omega, \quad j=1, \ldots, g .
$$

If $-\hat{\mathbf{B}}_{j}$ are rational, then $\mathfrak{K}$ is the least common multiple of the denominators of these national numbers. For example, if

$$
\hat{\mathbf{B}}=\left(\begin{array}{c}
-\frac{1}{3} \\
-\frac{1}{5} \\
-\frac{1}{6}
\end{array}\right),
$$

then $\mathfrak{K}=30$. However, if at least one of the components of the vector $\hat{\mathbf{B}}$ is an irrational number, then it is impossible to find an integer $\mathfrak{K}$ that satisfies condition (4.21). These correspond to the cases where the recurrence coefficients never repeat themselves or equivalently, the sets for which there is no polynomial mapping from this set onto $[-1,1]$.

Note that in general the values of $\hat{\mathbf{B}}_{j}$ are subject to certain conditions. In 9], section 10, a physical interpretation was given; the values $-\hat{\mathbf{B}}_{j}$ were seen to be the proportion of the overall charge lying on the interval $\left[\beta_{j-1}, \alpha_{j}\right]$, where $\beta_{0}:=-1$, in the equilibrium charge density for the set $E$, when the total charge was normalized to 1 , that is,

$$
\int_{E} \sigma(x) d x=1
$$


Since the total charge on all of the intervals must add up to 1 the following inequality is satisfied by the components of $\hat{\mathbf{B}}$ :

$$
0<\sum_{j=1}^{g}-\hat{\mathbf{B}}_{j}<1 .
$$

To finish this section we refer to figure 2. Recall that each point in the plane

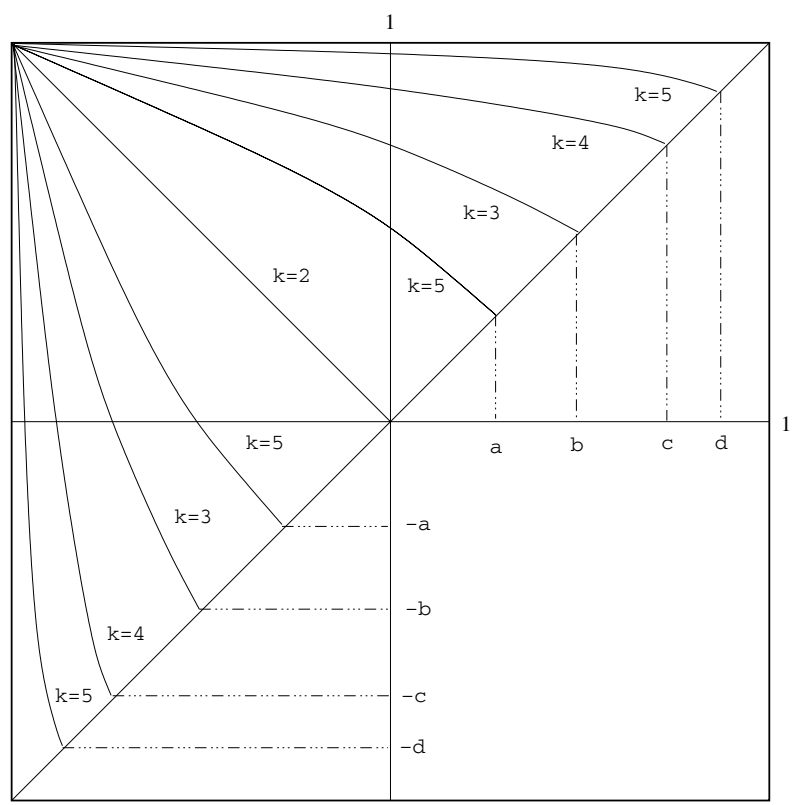

Figure 2. Contours of constant periodicity in the $(\alpha, \beta)$ plane for genus 1 . Here $a=(-1+\sqrt{5}) / 4, b=1 / 2, c=1 / \sqrt{2}$ and $d=(1+\sqrt{5}) / 4$. This is not a precise plot, it is representative of the relative position of the contours.

represents a configuration of the branch points for $g=1$ and therefore a sequence of orthogonal polynomials. Using our notation, if $\left\{\tilde{x}_{l}\right\}$ represents a curve in this plane with integer $\mathfrak{K}$, then $\left\{-x_{i}\right\}$ represents a different curve but with the same integer $\mathfrak{K}$. We ask the question "Is there a relationship between the polynomials associated with the curves $\left\{x_{i}\right\}$ and $\left\{-x_{i}\right\}$ ?" A similar question can also be asked for $g>1$. The relation between these cases is given in the most general form by theorem 1.2. However, since the recurrence coefficients are periodic, the elements appearing in the determinant simplify:

$$
P_{n \mathfrak{K}+j}\left(\beta_{l}\right)=\frac{1}{2} P_{n \mathfrak{K}}\left(\beta_{l}\right) P_{j}\left(\beta_{l}\right), \quad 1 \leq j \leq \mathfrak{K}-1,
$$

and

$$
Q_{n \mathfrak{K}+j}\left(\alpha_{l}\right)=\frac{1}{2} P_{n \mathfrak{K}}\left(\alpha_{l}\right) Q_{j}\left(\alpha_{l}\right), \quad 1 \leq j \leq \mathfrak{K}-1,
$$

where

$$
P_{n \mathfrak{K}}\left(\alpha_{l}\right)=P_{n \mathfrak{K}}\left(\beta_{l}\right)=\frac{\Delta_{\mathfrak{K}}^{n}(-1)^{n(l+\mathfrak{K})}}{2^{n-1}} .
$$


As an example we show the relation between the polynomials associated with the curves $\left\{-\frac{1}{2}\right\}$ and $\left\{\frac{1}{2}\right\}$ which are the two $\mathfrak{K}=3 \mathrm{~g}=1$ curves. Using the notation of theorem 1.2, we denote the polynomials associated with the set $\left\{-\frac{1}{2}\right\}$ as $P_{n}(x)$, the polynomials associated with the set $\left\{\frac{1}{2}\right\}$ as $\tilde{P}_{n}(x)$, and the respective mapping polynomials as $M_{3}(x)$ and $\tilde{M}_{3}(x)$. It is easy to verify that

$$
M_{3}(x)=(-1) \tilde{M}_{3}(-x) \text {. }
$$

Consequently,

$$
\tilde{P}_{3 n}(x)=(-1)^{3 n} P_{3 n}(-x) .
$$

However, for the intermediate polynomials we must use the expressions given in theorem 1.2 , which give

$$
\begin{aligned}
\tilde{P}_{3 n+1}(x)=\frac{(-1)^{3 n}}{\left(x+\alpha+2-4 \sqrt{\frac{\alpha+1}{2}}\right)} & {\left[P_{3 n+2}(-x)-\left(\sqrt{\frac{\alpha+1}{2}}-1\right) P_{3 n+1}(-x)\right.} \\
+ & \left.\left((\alpha+3) \sqrt{\frac{\alpha+1}{2}}-2(\alpha+1)\right) P_{3 n}(-x)\right],
\end{aligned}
$$

and

$$
\begin{aligned}
& \tilde{P}_{3 n+2}(x)=\frac{(-1)^{3 n+1}}{\left(x+\alpha+2-4 \sqrt{\frac{\alpha+1}{2}}\right)}\left[P_{3(n+1)}(-x)+\left(\sqrt{\frac{\alpha+1}{2}}\right) P_{3 n+2}(-x)\right. \\
& \left.-\frac{1}{2}\left(1+\alpha-2 \sqrt{\frac{1+\alpha}{2}}\right) P_{3 n+1}(-x)\right] \text {. }
\end{aligned}
$$

4b. General form of the polynomials. In this section we show some plots of the polynomials for small $\mathfrak{K}$ and prove a theorem regarding the zeroes. The Chebyshev polynomials $\widehat{T}_{n}(x)$ is bounded between 1 and -1 . These are the mapping polynomials from $[-1,1]$ to $[-1,1]$. As a consequence of (4.5) we see that $\left|\widehat{P}_{n \mathfrak{K}}(x)\right| \leq$ 1 for $x \in E$, where

$$
\widehat{P}_{n \mathfrak{K}}(x)=\frac{2^{n-1}}{\left(\Delta_{\mathfrak{K}}\right)^{n}} P_{n \mathfrak{K}}(x) .
$$

Outside of the set we no longer have this bound. Therefore we would expect the polynomials to appear graphically as in figure 3 . In figures 4,5 and 6 we plot some of the intermediate polynomials $\widehat{P}_{n \mathfrak{K}+j}(x)$ for various values of $j$ and $\mathfrak{K}$, where

$$
\widehat{P}_{n \mathfrak{K}+j}(x)=\frac{2^{n}}{\left(\Delta_{\mathfrak{K}}\right)^{n} \sqrt{2 h_{j}}} P_{n \mathfrak{K}+j}(x) .
$$

In $E$ they are bounded above and below by some function. It is clear from the representation (1.18) that the form of the envelope is $\pm \widehat{\rho}_{n \mathfrak{K}+j}(x)$, for $x \in E$, where

$$
\begin{aligned}
\widehat{\rho}_{n \mathfrak{K}+j}(x) & =\frac{2^{n}}{\left(\Delta_{\mathfrak{K})^{n} \sqrt{2 h_{j}}}\right.} \sqrt{\frac{S_{g}(x, n \mathfrak{K}+j)}{\prod_{l=1}^{g}\left(x-\alpha_{l}\right)}} \\
& =\sqrt{\frac{\prod_{j=1}^{g}\left(x-\gamma_{j}(n \mathfrak{K}+j)\right)}{\prod_{j=1}^{g}\left(x-\alpha_{j}\right)}} .
\end{aligned}
$$

Note that the quantity under the square root is strictly positive for $x \in E$. 


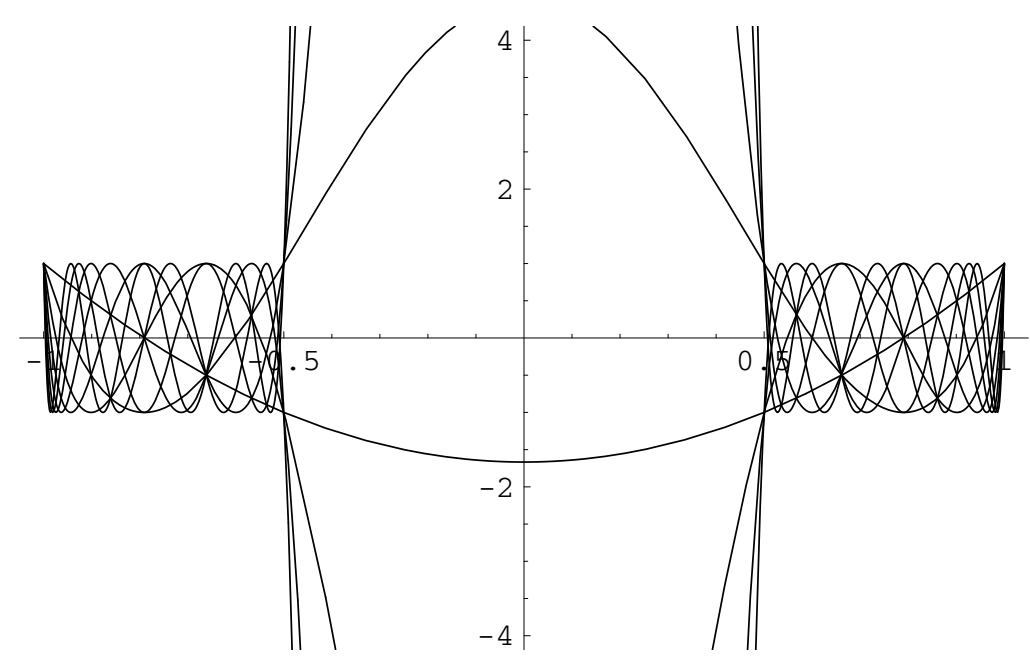

FiguRE $3 . \quad \mathfrak{K}=2, j=0, n=1 \ldots 8, \alpha=-0.5$

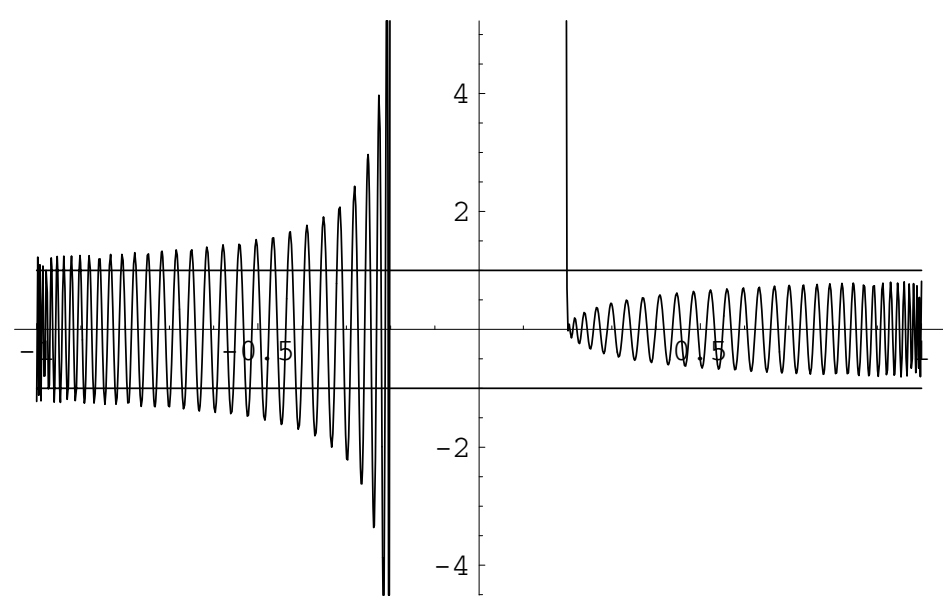

FiguRE $4 . \quad \mathfrak{K}=2, j=1, n=65, \alpha=-0.2$

We already know something about the zeros of the generalized chebyshev polynomials in the most general case from [9. In the polynomial mapping case we can say a little more about the zeros.

Theorem 4.2. All of the zeros of $P_{n \mathfrak{K}}(x)$ lie inside $E$, and in each of the $\mathfrak{K}$ subintervals that make up E lie $n$ zeros.

Proof. All of the zeros of the Chebyshev polynomials are contained in $(-1,1)$. From (4.5), and since $\frac{P_{\mathfrak{K}}(x)}{\Delta_{\mathfrak{K}}} \in[-1,1]$ if and only if $x \in E$, we see that all of the zeros of $P_{n \mathfrak{K}}(x)$ lie inside $E$ and each subinterval $E_{i}$ contains $n$ zeros.

The zeros of $Q_{n \mathfrak{K}}(x)$ behave similarly except in this case there are $n-1$ zeros in each interval $E_{i}$ and there are also zeros at every $\alpha_{j}, j=1, \ldots, \mathfrak{K}-1$. This is easily verified by checking the form of (4.6). From (4.6) the $n-1$ zeros in each $E_{i}$ come 


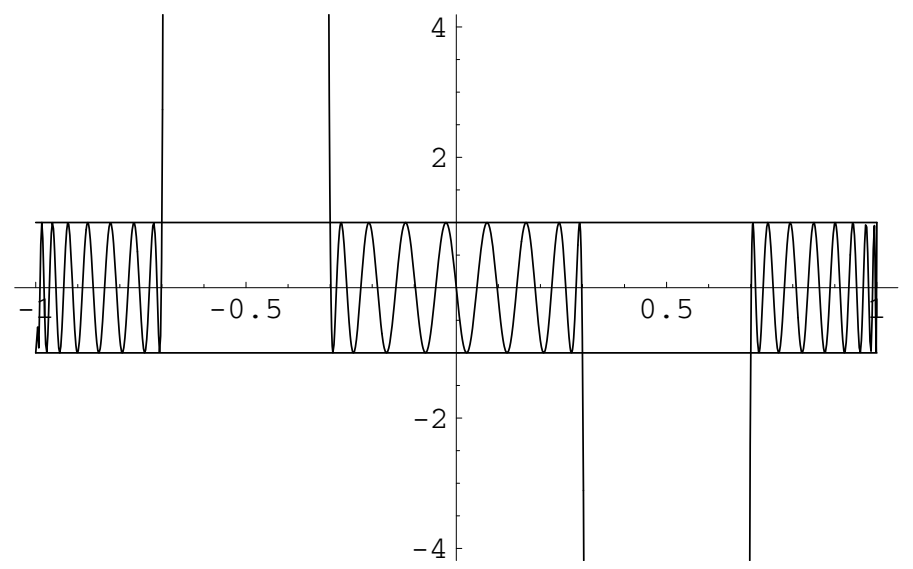

Figure 5. The symmetric case for $\mathfrak{K}=3, j=0, n=17, \alpha=-0.7$

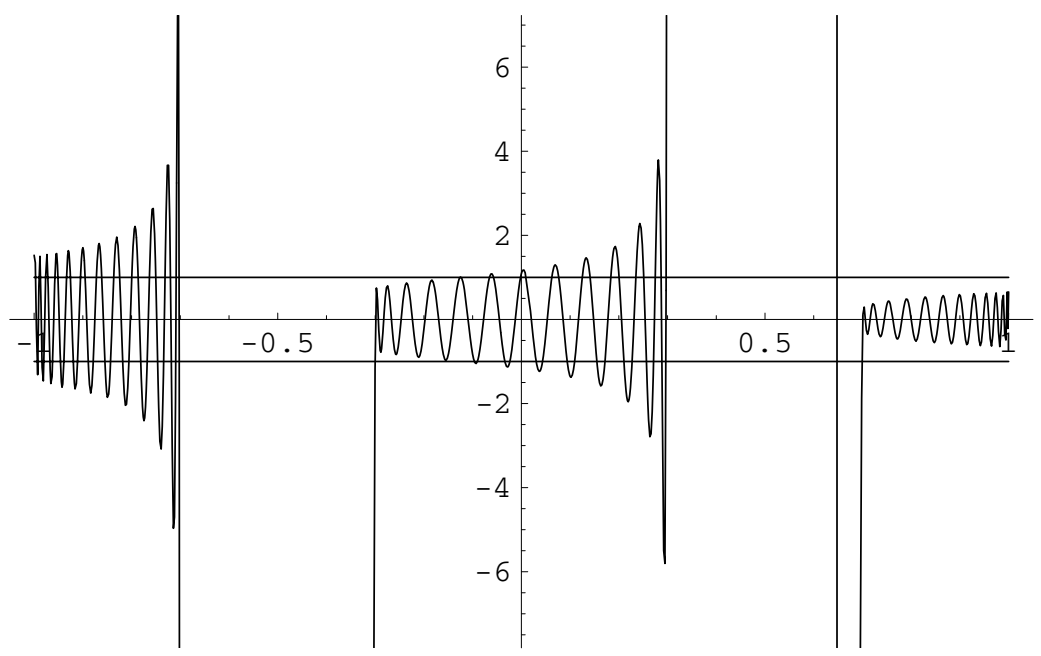

Figure 6 . The symmetric case for $\mathfrak{K}=3, j=1, n=25, \alpha=-0.7$

for the zeros of $U_{n}\left(\frac{P_{\mathfrak{K}}}{\Delta_{\mathfrak{K}}}\right)$. In each interval $E_{i}$, these zeros interlace with the zeros of $P_{n \mathfrak{K}}(x)$.

Theorem 4.3. Let $\left\{x_{i, n}\right\}$ denote the zeros of $P_{n}(x)$ and $\left\{y_{i, n}\right\}$ denote the zeros of $Q_{n}(x)$. Consider the following two sets:

$$
\begin{aligned}
& A:=\left\{\left\{x_{i, n \mathfrak{K}}\right\},\left\{x_{i, j}\right\}\right\}, \\
& B:=\left\{-1,1, \beta_{1} \ldots \beta_{\mathfrak{K}-1},\left\{y_{i, j}\right\},\left\{y_{i, n \mathfrak{K}}\right\}\right\} \backslash\left\{\alpha_{1} \ldots \alpha_{\mathfrak{K}-1}\right\} .
\end{aligned}
$$

The following three statements are true:

Between, any zero of $P_{j}(x)$, and any element of $B$ which is larger than this zero, there lies at least one zero of $P_{n \mathfrak{K}+j}(x)$.

Between any zero of $Q_{j}(x)$, and any element of $A$ which is larger than this zero there lies at least one zero of $P_{n \mathfrak{K}+j}(x)$. 
If $P_{j}(x)$ has a zero at any of the points in $B$, then $P_{n \mathfrak{K}+j}(x)$ has a zero at the same point.

Proof. From (4.7), $x_{i, n \mathfrak{K}+j}$ solves the following equation:

$$
P_{n \mathfrak{K}}(x) P_{j}(x)=-\frac{1}{\psi^{2}(x)} Q_{n \mathfrak{K}}(x) Q_{j}(x) .
$$

Using (4.5) and (4.6), we see that this is equivalent to

$$
\Delta_{\mathfrak{K}} T_{n}\left(\frac{P_{\mathfrak{K}}(x)}{\Delta_{\mathfrak{K}}}\right) P_{j}(x)=U_{n}\left(\frac{P_{\mathfrak{K}}(x)}{\Delta_{\mathfrak{K}}}\right) Q_{j}(x)\left(1-x^{2}\right) \prod_{i=1}^{\mathfrak{K}-1}\left(x-\beta_{i}\right) .
$$

For clarity, we illustrate a proof of the case of $\mathfrak{K}=3$ and $n=2$. The zeroes of $C(x)$ and $D(x)$, where

and

$$
C(x):=\Delta_{\mathfrak{K}} T_{n}\left(\frac{P_{\mathfrak{K}}(x)}{\Delta_{\mathfrak{K}}}\right)
$$

$$
D(x):=\left(1-x^{2}\right) U_{n}\left(\frac{P_{\mathfrak{K}}(x)}{\Delta_{\mathfrak{K}}}\right) \prod_{i=1}^{\mathfrak{K}-1}\left(x-\beta_{i}\right),
$$

are indicated in figure 7 . The crosses denote the zeros of $C(x)$ and the dots denote the zeros of $D(x)$. We see that they interlace. In order to plot the remaining

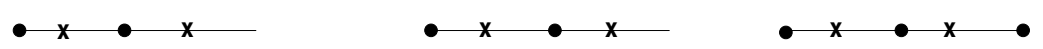

Figure 7 . The zeroes of $C(x)$ and $D(x)$ for $\mathfrak{K}=3$ and $n=2$. The three line segments are the disjoint intervals that make up $E$. The crosses denote the zeroes of $C(x)$ and the dots, the zeroes of $D(x)$.

zeros of (4.23) we must add $j$ crosses and $j-1$ dots, corresponding to the zeros of $P_{j}(x)$ and $Q_{j}(x)$ respectively. We can distribute these without any restriction other than they must also interlace with each other. The result is a sequence of crosses and dots that alternate except for $j$ occurrences of two adjacent crosses and $j-1$ occurrences of two adjacent dots. Where we see two adjacent crosses, one of them is a zero of $P_{j}(x)$. Similarly with two dots, one of them is a zero of $Q_{j}(x)$. As an example we plot where the zeroes of both sides of (4.23) might lie, for $\mathfrak{K}=3$, $n=2$ and $j=2$ in figure 8 .
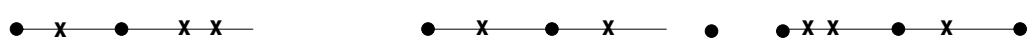

FiguRE 8.

In order to graph the polynomials that pass through the zeros we need an initial condition. The condition is that both sides of (4.23) have the same sign at $x=-1+\epsilon$ where $\epsilon$ is a small positive constant. This is true because close to $x=-1, P_{j}(x)$ and $Q_{j}(x)$ must have the opposite sign. Similarly, $T_{n}\left(\frac{P_{\mathfrak{K}}(x)}{\Delta_{\mathfrak{K}}}\right)$ and $U_{n}\left(\frac{P_{\mathfrak{K}}(x)}{\Delta_{\mathfrak{K}}}\right)$ have the same sign if $\mathfrak{K}$ is even and have the opposite sign if $\mathfrak{K}$ is odd. The product $\prod_{j=1}^{\mathfrak{K}-1}\left(x-\beta_{i}\right)$, for $x=-1+\epsilon$ is negative if $\mathfrak{K}$ is even and positive if $\mathfrak{K}$ is odd. $\Delta_{\mathfrak{K}}$ 
is a positive constant, and $\left(1-x^{2}\right)$ is also positive. Therefore both sides of (4.23) have the same sign at $x=-1+\epsilon$.

Therefore if we draw lines connecting the dots and the crosses that represent the tonicity of the polynomials we will see the pattern shown in figure 9 .

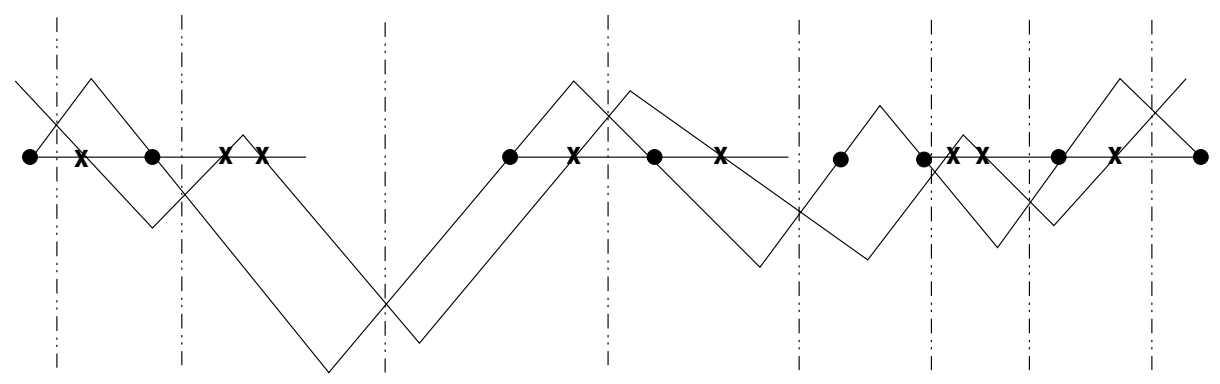

Figure 9. The intersections of the two lines represent qualitatively where the zeroes of $P_{n \mathfrak{K}+j}(x)$ will lie relative to the zeroes of both sides of (4.23)

We are interested in where these polynomials intersect, as these points represent the zeros of $P_{n \mathfrak{K}+j}(x)$. Starting from the point $x=-1$ and moving along the interval $[-1,1]$, the following behaviour is observed. Before the first occurrence of two adjacent crosses the polynomials intersect between every dot-cross pair. After the appearance of the two adjacent crosses, the polynomials intersect between every cross-dot pair. This continues until we reach the first occurrence of two adjacent dots, and then the intersection of the polynomials appears again in between every dot-cross pair. So every appearance of two crosses or two dots swaps the position of the intersection points between dot-cross pairs and cross-dot pairs. Since the appearance of two dots and two crosses alternate, after every appearance of two crosses there must be a point of intersection before the next dot. This corresponds to the statement in the theorem that between every zero of $P_{j}(x)$ and the next element from the set $B$ lies a zero of $P_{n \mathfrak{K}+j}(x)$. Similarly, after the appearance of two dots there must be an intersection point before the next cross. This is equivalent to saying that between every zero of $Q_{j}(x)$ and the next element from set $A$ there also lies a zero of $P_{n \mathfrak{K}+j}(x)$.

Corollary. If $P_{j}(x)$ has a zero in $\bar{E}_{i}$, then so does $P_{n \mathfrak{K}+j}(x)\left(\bar{E}_{i}:=\left(\alpha_{i}, \beta_{i}\right)\right)$.

Proof. Referring to figure 9 we see that there is always a dot at the beta points. If there is a zero of $P_{j}(x)$ in $\bar{E}_{i}$, then the previous theorem tells us that there must be a zero of $P_{n \mathfrak{K}+j}$ between this zero and the next $\beta$ point. Since $\bar{E}_{i}$ ends at the next $\beta$ point the zero of $P_{n \mathfrak{K}+j}$ is also contained in $\bar{E}_{i}$.

\section{Appendix A. Genus one Recurrence Coefficients}

Here we show one method of determining the recurrence coefficients for the genus 1 case explicitly in terms of the branch points $\alpha$ and $\beta$. It involves manipulating the theta function expressions given in (1.8) and (1.9). We see explicitly how the Jacobian elliptic functions define the form of the coefficients. For $g=1$ we have

$$
b_{n}=\frac{\beta-\alpha}{2}+\frac{1}{A}\left[\frac{\vartheta_{3}^{\prime}\left(3 u^{+}\right)}{\vartheta_{3}\left(3 u^{+}\right)}-\frac{\vartheta_{3}^{\prime}\left(u^{+}\right)}{\vartheta_{3}\left(u^{+}\right)}+\frac{\vartheta_{3}^{\prime}\left((2 n-1) u^{+}\right)}{\vartheta_{3}\left((2 n-1) u^{+}\right)}-\frac{\vartheta_{3}^{\prime}\left((2 n+1) u^{+}\right)}{\vartheta_{3}\left((2 n+1) u^{+}\right)}\right],
$$


where

$$
A=\frac{4 K}{\sqrt{(1-\alpha)(1+\beta)}}
$$

and

$$
u^{+}=\frac{1}{2 K} \int_{0}^{\sqrt{\frac{\beta+1}{2}}} \frac{d t}{\sqrt{\left(1-t^{2}\right)\left(1-k^{2} t^{2}\right)}}
$$

with

$$
k^{2}=\frac{2(\beta-\alpha)}{(1-\alpha)(1+\beta)} .
$$

Using

$$
\vartheta_{3}(v)=\vartheta_{0}\left(v \pm \frac{1}{2}\right)
$$

and

$$
\theta_{j}(v)=\vartheta_{j}\left(\frac{v}{2 K}\right)
$$

we write

$$
\begin{aligned}
b_{n}=\frac{\beta-\alpha}{2}+\frac{2 K}{A}\left[\frac{\theta_{0}^{\prime}\left(6 K u^{+}+K\right)}{\theta_{0}\left(6 K u^{+}+K\right)}-\frac{\theta_{0}^{\prime}\left(2 K u^{+}-K\right)}{\theta_{0}\left(2 K u^{+}-K\right)}\right. \\
\left.\quad+\frac{\theta_{0}^{\prime}\left((2 n-1) 2 K u^{+}-K\right)}{\theta_{0}\left((2 n-1) 2 K u^{+}-K\right)}-\frac{\theta_{0}^{\prime}\left((2 n+1) 2 K u^{+}+K\right)}{\theta_{0}\left((2 n+1) 2 K u^{+}+K\right)}\right] .
\end{aligned}
$$

The Jacobi function is defined to be

$$
Z(w)=\frac{\theta_{0}^{\prime}(w)}{\theta_{0}(w)}
$$

and it satisfies the following formula:

$$
Z(u+v)-Z(u-v)-2 Z(v)=\frac{-2 k^{2} \operatorname{sn}^{2}(u) \operatorname{sn}(v) \operatorname{cn}(v) \operatorname{dn}(v)}{1-k^{2} \operatorname{sn}^{2} u \operatorname{sn}^{2} v} .
$$

If we make the choice

$$
\begin{aligned}
u_{1} & =4 n K u^{+}, \\
u_{2} & =4 K u^{+}, \\
v & =2 K u^{+}+K,
\end{aligned}
$$

then we have

$$
b_{n}=\frac{\beta-\alpha}{2}+\frac{2 K}{A}\left[Z\left(u_{2}+v\right)-Z\left(u_{2}-v\right)+Z\left(u_{1}-v\right)-Z\left(u_{1}+v\right)\right] .
$$

After using the addition formula for $Z(v)$ and applying some basic identities $\left(k^{\prime}=\sqrt{1-k^{2}}\right)$,

$$
\begin{aligned}
& \operatorname{sn}(w+K)=\frac{\operatorname{cn}(w)}{\operatorname{dn}(w)} \\
& \operatorname{cn}(w+K)=-k^{\prime} \frac{\operatorname{sn}(w)}{\operatorname{dn}(w)} \\
& \operatorname{dn}(w+K)=\frac{-k^{\prime}}{\operatorname{dn}(w)}
\end{aligned}
$$


and

$$
\begin{aligned}
\operatorname{sn}^{2} w+\operatorname{cn}^{2} w & =1, \\
k^{2} \operatorname{sn}^{2} w+\operatorname{dn}^{2} w & =1,
\end{aligned}
$$

we can write,

$b_{n}=\frac{\beta-\alpha}{2}+(\beta-\alpha)(1+\alpha)\left[\frac{\operatorname{sn}^{2}\left(4 n K u^{+}\right)}{(1+\beta)-(\beta-\alpha) \operatorname{sn}^{2}\left(4 n K u^{+}\right)}-\frac{4(1-\alpha)}{(\beta+\alpha)^{2}+4\left(1-\alpha^{2}\right)}\right]$

since

$$
\operatorname{sn}\left(2 K u^{+}\right)=\sqrt{\frac{\beta+1}{2}} .
$$

We have the following addition formula for $\operatorname{sn}(u)$ valid for any $u$ and $v$ :

$$
\operatorname{sn}(u+v)=\frac{\operatorname{sn}(u) \operatorname{cn}(v) \operatorname{dn}(v)+\operatorname{sn}(v) \operatorname{cn}(u) \operatorname{dn}(u)}{1-k^{2} \operatorname{sn}^{2}(u) \operatorname{sn}^{2}(v)} .
$$

We can iterate this to obtain $\operatorname{sn}\left(4 n K u^{+}\right)$in terms of $k$ and $\operatorname{sn}\left(2 K u^{+}\right)$. This gives us in principle, a method for evaluating the recurrence coefficients in terms of $\alpha$ and $\beta$. Owing to the nature of the addition formula, after just a few iterations the expressions for the coefficients become very large.

The $a_{n}$ are given as

$$
a_{n}=\left[\frac{\vartheta_{1}^{\prime}(0)}{A \vartheta_{1}\left(2 u^{+}\right)}\right]^{2} \frac{\vartheta_{3}^{2}\left((2 n-1) u^{+}\right)}{\vartheta_{3}\left((2 n+1) u^{+}\right) \vartheta_{3}\left((2 n-3) u^{+}\right)}, \quad n \geq 2 .
$$

Using the following identity:

$$
1-k^{2} \operatorname{sn}^{2}(u) \operatorname{sn}^{2}(v)=\theta_{0}(0)^{2} \frac{\theta_{0}(u+v) \theta_{0}(u-v)}{\theta_{0}^{2}(u) \theta_{0}^{2}(v)},
$$

with

$$
\begin{aligned}
& u=(2 n-1) 2 K u^{+}+K, \\
& v=4 K u^{+}
\end{aligned}
$$

we have

$$
\begin{aligned}
a_{n} & =c \frac{1}{\left(1-k^{2} \operatorname{sn}^{2}\left((2 n-1) 2 K u^{+}+K\right) \operatorname{sn}^{2}\left(4 K u^{+}\right)\right)}, \quad n \geq 2 \\
& =c \frac{1}{\left(1-\frac{8(\beta-\alpha)}{(2-\alpha+\beta)^{2}} \operatorname{sn}^{2}\left((2 n-1) 2 K u^{+}+K\right)\right)} .
\end{aligned}
$$

Here $c$ is a constant independent of $n$,

$$
c=\left(\frac{\vartheta_{1}^{\prime}(0) \vartheta_{0}(0)}{A \vartheta_{0}\left(2 u^{+}\right) \vartheta_{1}\left(2 u^{+}\right)}\right)^{2} .
$$

To determine $c$ explicitly, we evaluate $a_{2}$ by comparing coefficients of (1.13) for $n=2$ and then compare this with $a_{2}$ given above. In which case we find

$$
c=\frac{\left[(\beta+\alpha)^{2}+4\left(1-\alpha^{2}\right)\right]^{2}}{16(2-\alpha+\beta)^{2}} .
$$

Note that in the higher genus cases, the non-linear difference equations derived in section 2 can be used to evaluate the recurrence coefficients. 


\section{Appendix B. Genus two auxiliary polynomials}

Using the method outlined in section 2 for $g=2$ we find

$$
\begin{aligned}
\frac{G_{3}(x, n)}{h_{n-1}}= & x^{3}-\frac{1}{2}\left(\alpha_{1}+\alpha_{2}+\beta_{1}+\beta_{2}\right) x^{2} \\
- & \left(\frac{1}{2}+\frac{\left(\alpha_{1}^{2}+\alpha_{2}^{2}+\beta_{1}^{2}+\beta_{2}^{2}\right)}{8}\right. \\
& \left.-\frac{1}{4}\left(\beta_{1} \beta_{2}+\alpha_{2} \beta_{1}+\alpha_{2} \beta_{2}+\alpha_{1} \alpha_{2}+\alpha_{1} \beta_{1}+\alpha_{1} \beta_{2}\right)-2 a_{n}\right) x \\
& +\frac{1}{16}\left(-\left(\alpha_{1}^{3}+\alpha_{2}^{3}+\beta_{1}^{3}+\beta_{2}^{3}\right)+4\left(\alpha_{1}+\alpha_{2}+\beta_{1}+\beta_{2}\right)\right. \\
& +\beta_{1} \beta_{2}\left(\beta_{1}+\beta_{2}\right)+\alpha_{2} \beta_{1}\left(\alpha_{2}+\beta_{1}\right)+\alpha_{2} \beta_{2}\left(\alpha_{2}+\beta_{2}\right)+\alpha_{1} \alpha_{2}\left(\alpha_{1}+\alpha_{2}\right) \\
& +\alpha_{1} \beta_{1}\left(\alpha_{1}+\beta_{1}\right)+\alpha_{1} \beta_{2}\left(\alpha_{1}+\beta_{2}\right) \\
& -2\left(\alpha_{2} \beta_{1} \beta_{2}+\alpha_{1} \beta_{1} \beta_{2}+\alpha_{1} \alpha_{2} \beta_{1}+\alpha_{1} \alpha_{2} \beta_{2}\right) \\
& \left.-16 a_{n}\left(\alpha_{1}+\alpha_{2}+\beta_{1}+\beta_{2}-2\left(b_{n}+b_{n+1}\right)\right)\right), \quad n \geq 2,
\end{aligned}
$$

and

$$
\begin{gathered}
\frac{S_{2}(x, n)}{h_{n}}=2 x^{2}-\left[\left(\alpha_{1}+\alpha_{2}+\beta_{1}+\beta_{2}\right)-2 b_{n+1}\right] x \\
-\frac{1}{4}\left(4+\alpha_{1}^{2}+\beta_{1}^{2}+\alpha_{2}^{2}+\beta_{2}^{2}-2\left(\alpha_{1} \alpha_{2}+\alpha_{1} \beta_{1}+\alpha_{2} \beta_{1}+\alpha_{1} \beta_{2}+\alpha_{2} \beta_{2}+\beta_{1} \beta_{2}\right)\right. \\
\left.-8\left(a_{n+1}+a_{n}\right)+4 b_{n+1}\left(\alpha_{1}+\alpha_{2}+\beta_{1}+\beta_{2}\right)-8 b_{n+1}^{2}\right), \quad n \geq 1, \\
G_{3}(x ; 1)=\left(x-b_{1}\right)\left(x-\alpha_{1}\right)\left(x-\alpha_{2}\right)
\end{gathered}
$$

and

$$
S_{2}(x ; 0)=\left(x-\alpha_{1}\right)\left(x-\alpha_{2}\right) .
$$

Incidentally, this gives us the $\gamma_{1,2}(n)$ coefficients for the genus 2 case as

$$
\begin{aligned}
\gamma_{1,2}(n) & =\frac{1}{4}\left(\alpha_{1}+\alpha_{2}+\beta_{1}+\beta_{2}\right)-2 b_{n+1} \\
& \mp\left(8+3\left(\alpha_{1}^{2}+\alpha_{2}^{2}+\beta_{1}^{2}+\beta_{2}^{2}\right)-2\left(\alpha_{1} \alpha_{2}+\alpha_{1} \beta_{1}+\alpha_{2} \beta_{1}+\alpha_{1} \beta_{2}+\alpha_{2} \beta_{2}+\beta_{1} \beta_{2}\right)\right. \\
& \left.-16\left(a_{n}+a_{n+1}\right)+4 b_{n+1}\left(\alpha_{1}+\alpha_{2}+\beta_{1}+\beta_{2}\right)-12 b_{n+1}^{2}\right)^{\frac{1}{2}}, \quad n \geq 1
\end{aligned}
$$

\section{REFERENCES}

1. N. I. Ahiezer, Orthogonal polynomials on several intervals, translated in Soviet Math. 1 (1960), 989-992. MR0110916 (22:1784)

2. N. I. Ahiezer, The Classical Moment Problem and Some Related Questions in Analysis, English translation, Oliver \& Boyed, Edinburgh, 1965. MR0184042 (32:1518)

3. N. I. Ahiezer and Ju. Ja. Tomčuk, On the theory of orthogonal polynomials over several intervals. (Russian) Dokl. Akad. Nauk USSR 138 (1961), 743-746. MR0131005 (24:A859)

4. N. I. Akhiezer, Elements of the Theory of Elliptic Functions (Transl. Math. Monographs vol. 79), Providence, RI: Amer. Math. Soc., 1990. MR1054205 (91k:33016) 
5. W. Al-Salam, W. Allaway, and R. Askey, Sieved ultraspherical polynomials, Trans. Amer. Math. Soc. 284 (1984), 39-55. MR0742411 (85j:33005)

6. J. A. Charris and M. E. H. Ismail, On sieved Orthogonal Polynomials II : Randon walk polynomials, Canad. J. Math. 38 (1986) 397-415. MR0833576 (87j:33014a)

7. J. A. Charris, M. E. H. Ismail, and S. Monsalve, On sieved Orthogonal Polynomials X : General Blocks of Recurrence Relations, Pacific J. Math. 163 (1994) 237-267. MR1262296 (95d:33004)

8. Y. Chen and M. E. H. Ismail, Ladder operators and differential equations for orthogonal polynomials, J. Phys. A 30 (1997), 7818-7829. MR1616931 (2000i:33011)

9. Y. Chen and N. Lawrence, A generalization of the Chebyshev polynomials, J. Phys. A 35 (2002) 4651-4699 (2001). MR1908638(2003k:33014)

10. J.S. Geronimo and W. Van Assche, Orthogonal polynomials on several intervals via a polynomial mapping, Trans. Amer. Math. Soc. 308 (1988) 559-581. MR0951620 (89f:42021)

11. Ya. L. Geronimus, On some finite difference equations and corresponding systems of orthogonal polynomials, Mem. Math. Sect. Fac. Phys. Kharkov State Univ. Kharkov Math. Soc. 25 (1975), 81-100.

12. M. E.H. Ismail, On sieved orthogonal polynomials III: Polynomials orthogonal on several intervals, Trans. Amer. Math. Soc. 294 (1986), 89-111. MR0819937 (87j:33014b)

13. M. E.H. Ismail, Discriminants and functions of the second kind for orthogonal polynomials, Results in Mathematics 34 (1998), 132-149. MR.1635590 (99g:42032)

14. F. Peherstorfer, On Bernstein-Szegő Orthogonal Polynomials on Several Intervals II: Orthogonal Polynomials with Periodic Recurrence Coefficients. J. Approx. Theo. 64 (1991) 123-161. MR 1091466 (92a:42031)

15. F. Peherstorfer and K. Schiefermeyr, Decription of Extremal Polynomials on Several Intervals and their Computation. I Acta. Math. Hungar. 83 (1999) 27-58. MR.1682902 (99m:41010)

16. G Szegö, Orthogonal Polynomials American Mathematical Society (1975). MR0372517 $(51: 8724)$

17. Ju. Ja. Tomčuk, Orthogonal Polynomials Over a System of Intervals on the Number Line, Zap. Fiz.-Mat. Khar'kov Mat. Oshch., 29 (1964) 93-128 (in Russian).

18. V. B. Uvarov, On the connection between polynomials, orthogonal with different weights, Dokl. Acad. Nauk SSSR, 126 (1959) 33-36. MR0149187 (26:6679)

19. V.B. Uvarov, The connection between systems of polynomials that are orthogonal with respect to different distribution functions, USSR Computat. Math. and Math. Phys. 9 (1969) 25-36. MR 0262764 (41:7369)

Department of Mathematics, Imperial College, London SW7 2BZ, United Kingdom

E-mail address: y.chen@ic.ac.uk

Department of Mathematics, University of Central Florida, Orlando, Florida 32826

E-mail address: jgriffin@math.ucf.edu

Current address: Department of Mathematics, American University of Sharjah, P.O. Box 26666, United Arab Emirates

E-mail address: jgriffin@aus.edu

Department of Mathematics, University of Central Florida, Orlando, Florida 32826

E-mail address: ismail@math.ucf.edu 